A N N A L E S Annales de Bretagne et des Pays de l'Ouest

Anjou. Maine. Poitou-Charente. Touraine

116-4 | 2009

Varia

\title{
Les Poitevins à la Chambre des comptes de Bretagne au XVIII ${ }^{\mathrm{e}}$ siècle
}

\author{
Guillaume Porchet
}

\section{OpenEdition}

Journals

Édition électronique

URL : http://journals.openedition.org/abpo/635

DOI : 10.4000/abpo.635

ISBN : 978-2-7535-1516-1

ISSN : 2108-6443

Éditeur

Presses universitaires de Rennes

Édition imprimée

Date de publication : 30 décembre 2009

Pagination : $91-121$

ISBN : 978-2-7535-1064-7

ISSN : 0399-0826

Référence électronique

Guillaume Porchet, "Les Poitevins à la Chambre des comptes de Bretagne au xvIII siècle », Annales de Bretagne et des Pays de l'Ouest [En ligne], 116-4 | 2009, mis en ligne le 30 décembre 2010, consulté le 23 septembre 2020. URL : http://journals.openedition.org/abpo/635 ; DOI : https://doi.org/10.4000/ abpo.635 


\title{
Les Poitevins à la Chambre des comptes de Bretagne au XVIII ${ }^{\mathrm{e}}$ siècle
}

\author{
Guillaume PORCHET \\ Étudiant de master, université de Nantes ${ }^{1}$
}

\begin{abstract}
"Quend à mon office de conseiller correcteur en la Chambre des comptes de Bretagne, pour luy trouver sans réplique daventage combiens je crois flaté plus deux mots, qu'avec votre aprobation Monsieur votre fils me comptanter l'estimer, quoique je sois en estat de vous prouver Messieurs meme par écrit incontestablement que depuy plus de douze ans, celles semblables [les charges de correcteur] qui se sont trouvées en ce cas destre vendues non par mort sont estés de trente quatre a trente six [mille livres]. Elles ont chacunes des gages sur létat du roy douze cent livres bien payées les trois termes par ans et en outre soixente dix huit livres tous les ans d'antrée et d'ailleurs sans compter tous les ans quelques réceptions d'officiers présidents généraux des finences encore soixante douze livres 16 sols. Nous y sommes bien cens offisiers majeurs sy acquérant la noblesse [...] avec pour nous nos enfans non establis tous les privillèges, préminences, exemptions et tous autres droits utilles honorables et aventageux de la premiere noblesse de lettre $[\ldots]^{2}$."
\end{abstract}

L'homme qui présente ainsi son office de correcteur est le poitevin Jean Victor Gabriel Chauvière, sieur de la Pagerie, qui l'exerce depuis 1763. Sans héritier mâle, il tente à la fin de l'année 1784 de convaincre son futur gendre et le père de ce dernier d'accepter pour dot sa charge anoblissante en plus de quelques domaines. Pour cela, il met l'accent sur les revenus et les exemptions procurés par sa charge ainsi que sur la noblesse graduelle qu'elle confère; il donne ainsi un rapide aperçu des raisons qui poussent bon nombre de familles à acquérir un office à la Chambre des comptes de Bretagne. Issus depuis le $\mathrm{XVI}^{\mathrm{e}}$ siècle du comté nantais, de la Bretagne et de l'Anjou, les magistrats se recrutent de plus en plus, au XvIII siècle,

1. Auteur d'un mémoire de Master 1 intitulé Les Poitevins à la Chambre des comptes de Bretagne, réalisé sous la direction de Dominique Le Page, université de Nantes, 2007, 2 tomes; et d'un mémoire de Master 2 intitulé Destins de quatre familles poitevines de la Chambre des comptes de Bretagne au XVIII siècle, réalisé sous la direction de Guy Saupin et de Dominique Le Page, université de Nantes, 2008.

2. Arch. dép. de la Vienne, J 140. 
en Poitou. À partir de la venue de Pierre I Rochard de Landebergère en 1702 jusqu'à la suppression de la chambre en 1791, pas moins de quarante Poitevins achètent un office pour servir dans cette cour. Le contraste est particulièrement net avec le siècle précédent, où seulement six Poitevins l'avaient intégrée alors que le $\mathrm{Xvl}^{\mathrm{e}}$ siècle en avait vu cinq. Pourquoi cette venue massive dans une cour souveraine jugée en crise par certains historiens? Qui sont ces nouveaux magistrats, d'où sont-ils originaires et que viennent-ils y chercher?

Pour répondre à ces différentes interrogations, je présenterai d'abord les origines sociales et géographiques des Poitevins, puis j'évoquerai les parcours qui les ont menés à la Chambre des comptes. Je m'intéresserai enfin à l'intérêt que pouvait représenter pour eux l'achat d'un office dans une cour souveraine tant sur le plan financier que social.

\section{Les origines des officiers}

Afin de bien cerner le profil des officiers poitevins qui ont réussi à intégrer la Chambre des comptes de Bretagne au XVIII ${ }^{\mathrm{e}}$ siècle, il convient de reconstituer autant que faire se peut leur environnement social.

\section{Une entrée groupée}

L'entrée des Poitevins à la Chambre des comptes de Bretagne débute en 1701 avec la vente par Jacques II Jaudonnet de Lavau-Richer de son office d'auditeur à Pierre I Rochard de Landebergère, tous deux vraisemblablement cousins ${ }^{3}$. Ce dernier, reçu à la Chambre en 1702, est à l'origine de la majorité des Poitevins originaires du sud du Bas-Poitou qui se succèdent dans la seconde cour souveraine de Bretagne jusqu'en 1786. Apparenté à de nombreuses familles bourgeoises de Fontenay-le-Comte et des environs par son mariage en 1708 avec Renée Marguerite Courtin, la fille d'un magistrat de la capitale bas poitevine, il sert alors de protecteur à un allié de sa belle-famille en la personne de Louis Cicoteau, sieur de la Touche. Celui-ci favorise à son tour la venue de parents, d'alliés et d'amis ${ }^{4}$. Les officiers du nord du Bas-Poitou (c'est-à-dire ceux originaires de la région qui va de Machecoul à Châtillon-sur-Sèvre ${ }^{5}$ en passant par Montaigu et Mortagne) moins nombreux ${ }^{6}$ se trouvent aussi liés entre eux par différents jeux d'alliance et sont également connectés avec leurs collègues de la basse province.

3. Si l'on croit la déclaration d'ancêtres que donne Jacques I Jaudonnet en 1645, son arrière-grand-mère paternelle serait née Frouin, et la mère de Pierre I Rochard s'appelait Perrine Frouin. Il y aurait donc des chances qu'ils aient été cousins.

4. Entre autres, Louis Cicoteau aide son ami Jude Badereau en 1724, originaire de Montaigu comme lui, à intégrer la Chambre. C'est même lui qui traite de son office d'auditeur.

5. Aujourd'hui Mauléon.

6. Par exemple, la famille Rocquand est originaire de Machecoul, la famille Badereau de Montaigu et la famille Boutillier de Mortagne. 
La présence des Poitevins à la Chambre des comptes s'accroît tout au long du XVIII ${ }^{\mathrm{e}}$ siècle, surtout à partir des années 1750. Ils finissent par composer une véritable " compagnie " au sein de la Chambre. Ceci est particulièrement visible lors de l'ouverture des semestres. À celle de septembre 1776, on trouve ainsi le président de Chassenon, les maîtres Robert et Panou de Faymoreau, le correcteur Chauvière, les auditeurs "Babin aîné et Babin cadet ", Arnault et le greffier en chef Cardin ${ }^{7}$. Tous ces magistrats se connaissent fort bien, d'autant plus que certains d'entre eux sont parents ou ont entretenu auparavant des relations professionnelles.

La perpétuation de cette présence poitevine s'explique aussi par le fait que la transmission des offices de la Chambre des comptes s'est opérée entre les Poitevins eux-mêmes (père/fils, alliés, etc.). Sur les 39 officiers connus qui vendent leurs offices à des Poitevins, 44 \% sont eux-mêmes d'origine poitevine et sur les 26 officiers poitevins qui ont pu vendre leurs offices avant la Révolution, 62 \% les cèdent à des personnes originaires du Poitou. Ces magistrats ont une nette préférence pour leurs familles et leurs alliés. Une fois qu'un office est entré dans un cercle familial, il a de fortes chances d'y rester. Cela explique également que les Poitevins détiennent surtout des offices subalternes, comme le montre le tableau ci-dessous où l'on constate qu'ils sont majoritairement auditeurs, correcteurs et greffiers, c'est-à-dire qu'ils ne siègent pas au bureau de la Chambre, là où se prennent les principales décisions.

Tableau 1 - Répartition des offices possédés par les Poitevins à la Chambre des comptes de Bretagne au XVII siècle

\begin{tabular}{|c|c|c|c|c|c|c|}
\hline Offices & $\begin{array}{c}\text { Greffiers } \\
\text { en chef }\end{array}$ & Auditeurs & Correcteurs & Maîtres & Présidents & Total \\
\hline Effectif & 6 & 24 & 3 & 6 & 2 & 41 \\
\hline
\end{tabular}

Nota : Duval de Chassenon fut maître puis président. Il est donc compté deux fois.

Proximité oblige, c'est le Bas Poitou qui fournit l'essentiel des magistrats alors qu'un seul officier du Haut Poitou intègre la Chambre, qui plus est en $1785^{8}$. Plus précisément, le recrutement s'opère dans les principaux centres urbains de la basse province : Fontenay-le-Comte, la capitale bas-poitevine, Luçon, le siège de l'évêché et les Sables-d'Olonne, le port atlantique. Les juridictions dont elles sont le siège offrent la possibilité aux familles qui en ont les moyens d'intégrer le monde des offices et d'en

7. Arch. dép. de Loire-Atlantique, B 673. Jean-Victor Chauvière de la Pagerie, correcteur de 1763 à 1785; Paul-François Babin des Ardilliers, auditeur de 1740 à 1778; MathurinJoseph Babin de la Chevallerie, auditeur de 1751 à 1779; Maximilien Arnault de la Motte, auditeur de 1769 à 1789 et Jean Cardin, greffier en chef de 1769 à 1790 .

8. Le Haut Poitou et le Bas-Poitou correspondent grosso modo aux départements de la Vienne et de la Vendée. Le Moyen-Poitou, qui n'existait pas en tant que tel, correspond aux Deux-Sèvres. 
gravir progressivement les principaux échelons. C'est toutefois Fontenayle-Comte qui est la ville d'origine de la majeure partie des officiers grâce aux cinq juridictions qu'elle abrite : pour près d'un tiers les officiers y sont nés et neuf autres magistrats y ont exercé un office ou y ont pris femme. En tout, un peu plus de la moitié des officiers ont été en rapport avec la capitale bas-poitevine.

La proximité évidente de Nantes joue également en faveur de Machecoul, Montaigu, Mortagne, voire Châtillon-sur-Sèvre. Même si ces cités ne concentrent que quelques milliers d'habitants tout au plus, elles constituent du fait de leur armature administrative (élections, traites foraines, greniers à sel, etc.) des relais utiles pour les familles de la région désirant faire carrière.

Carte 1 - Origines géographiques des quarante officiers poitevins de la Chambre des comptes de Bretagne au XVIII siècle

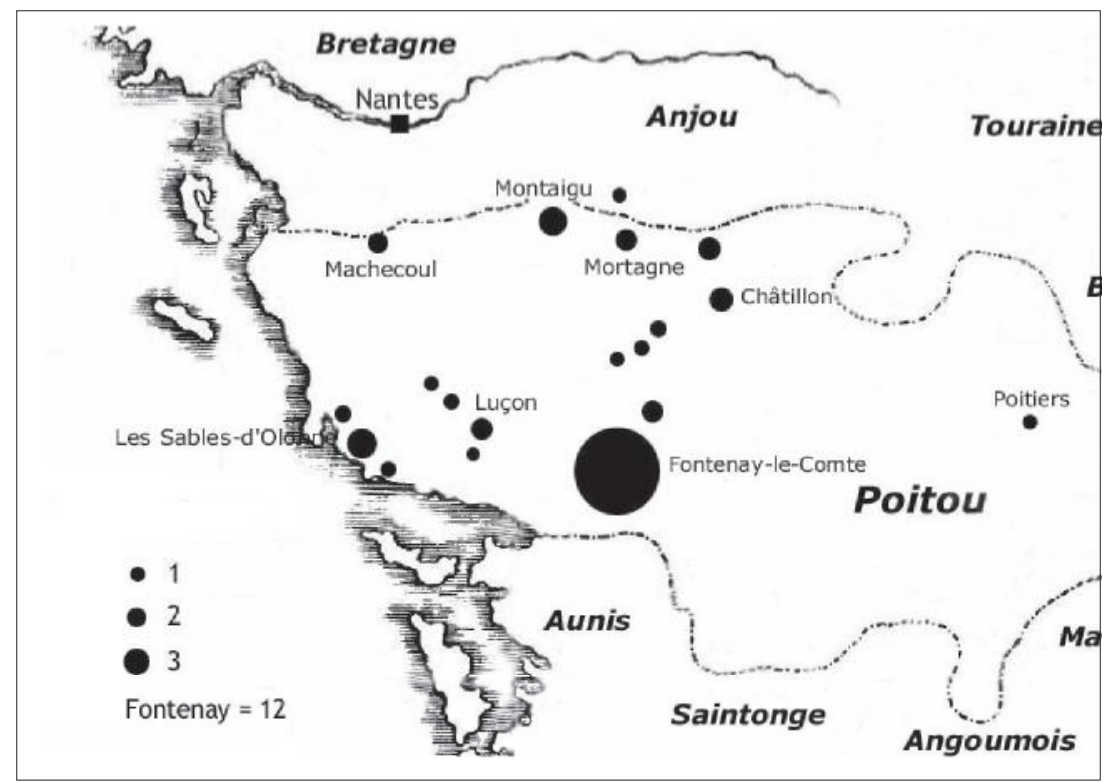

Il est intéressant de souligner que les trois villes du Bas-Poitou possédant un siège d'élection, en l'occurrence Fontenay, les Sables et Châtillon, sont aussi les principales aires de recrutement. Les juridictions inférieures des finances paraissent ainsi être des tremplins vers les offices de la Chambre des comptes. Pourtant, il est surprenant que Poitiers n'offre qu'un officier et Niort, siège d'une élection et seconde ville poitevine par son importance démographique, n'en donne aucun. Niort semble être tournée beaucoup plus vers le commerce que les offices et Poitiers possède déjà 
un Bureau des finances dont les offices confèrent la noblesse ${ }^{9}$. Sans doute aussi les élites de ces deux villes se sont-elles orientées vers d'autres pôles comme Paris, Rennes ou Bordeaux.

Si leurs origines géographiques semblent très homogènes, qu'en est-il de leurs origines sociales?

\section{Les origines sociales}

Tableau 2 - Les professions des pères, aïeux et bisä̈eux paternels

\begin{tabular}{|l|c|c|c|}
\hline \multirow{2}{*}{\multicolumn{1}{|c|}{ Milieu social d'origine }} & \multicolumn{3}{|c|}{ Part en \% } \\
\cline { 2 - 4 } & Pères & Aïeux & Bisaïeux \\
\hline Chambre des comptes & 36 & - & - \\
\hline Officiers de finance & 3 & 9 & 7 \\
\hline Officiers de justice & 22 & 22 & 21 \\
\hline Officiers ministériels & 18 & 24 & 31 \\
\hline Médecins & 5 & 3 & - \\
\hline Propriétaires rentiers & 3 & 8 & 11 \\
\hline Négociants, marchands & - & 8 & 15 \\
\hline Fermiers seigneuriaux & - & 3 & 11 \\
\hline Indécis & 13 & 23 & 400 \\
\hline \multicolumn{1}{|c|}{ Total } & 100 & 100 & \\
\hline
\end{tabular}

Ces pourcentages, obtenus d'après 67,5\% des professions connues pour les bisaïeux, 87,5 \% pour les grands-pères et $97,5 \%$ pour les pères ${ }^{10}$, permettent d'apprécier les origines sociales des vingt-deux familles poitevines.

Les recherches menées sur les bisaïeux paternels révèlent que pour au moins un quart d'entre eux ils ont été des fermiers seigneuriaux et des marchands ${ }^{11}$. Le commerce a majoritairement été le moteur de l'ascension sociale des familles de Fontenay-le-Comte ou des Sables-d'Olonne, à l'exemple de la famille de l'auditeur Jannet dont le plus lointain ancêtre connu était marchand à la Chaume au Xvle siècle. Certaines familles, voire certains magistrats, n'ont d'ailleurs pas rompu tout lien avec le monde commercial; ainsi en va-t-il pour le greffier en chef Jean Biaille de Lengibaudière. Le père de ce dernier, qui avait été procureur fiscal de la commanderie de Champgillon, pratiquait en même temps le commerce sur mer. De même,

9. Des alliés, des cousins, voire des frères de ces officiers se sont engagés dans le Bureau des finances de Poitiers au cours du XVIII ${ }^{\mathrm{e}}$ siècle, comme François-Alexandre Duval de la Vergne, le frère cadet du Président François Gabriel Duval de la Vergne, qui acheta en 1743 un office de trésorier.

10. Seule la profession du père de l'auditeur Pierre I Rochard reste inconnue.

11. L'ancêtre de l'auditeur Panou de Faymoreau, par exemple, était marchand à Niort à la fin du XVI ${ }^{\mathrm{e}}$ siècle et la génération suivante a continué sur la même voie en associant de petits offices royaux et la régie de terres seigneuriales. 
Jean Biaille, alors greffier en chef, s'était trouvé fondé vers 1750 " pour un seize dans un navire [mais] le succès en fut si peu heureux qu'il ne retira même pas les fonds " mais plus tard il fit valoir l'héritage de son père " dans le commerce pendant 22 années ${ }^{12}$ ». Ces initiatives commerçantes, plus ou moins assidues, prouvent que les officiers d'une cour souveraine pouvaient très bien placer de l'argent dans le négoce malgré le mépris éprouvé par les élites nobiliaires pour ce genre de pratique ${ }^{13}$.

Au moins $11 \%$ de leurs bisaïeux se sont occupé de fermes seigneuriales, en cumulant d'ailleurs souvent cette profession avec un office de judicature ou de finance, comme l'a fait l'arrière-grand-père du maître aux comptes Robert de la Levraudière, fermier seigneurial de la Boissière et receveur du chapitre de Luçon, ou avec une fonction médicale ${ }^{14}$.

Malgré ces carrières professionnelles où le maniement d'argent était important, les recherches sur leurs ancêtres montrent que ces familles étaient établies depuis plusieurs générations déjà dans le monde de l'office. Pour plus des deux tiers en effet, ces familles sont issues du monde de la judicature et de la finance, et plus précisément des petites juridictions seigneuriales et royales. Les sièges royaux, les sénéchaussées ou les élections sont sur-représentés. Les officiers ministériels (procureurs, notaires ou greffiers) qu'ils soient royaux ou seigneuriaux ne sont pas non plus à négliger. Les familles du monde de la judicature sont plus nombreuses que celles du monde de la finance, même si certaines d'entre elles ont joué sur les deux tableaux pour assurer leur ascension sociale ${ }^{15}$. Seule la famille Cossin, noble depuis le $\mathrm{xvl}^{\mathrm{e}}$ siècle, avait des ancêtres militaires, mais ces derniers avaient également exercé des charges de judicature.

Qu'en est-il de la politique matrimoniale de ces magistrats? On sait que sous l'Ancien Régime, le mariage était avant tout une affaire d'intérêt qui tenait compte de l'honorabilité, de la fortune et de l'ancienneté de la maison de l'épouse. Sur les quarante officiers poitevins, seulement quatre sont décédés sans avoir contracté de mariage, deux étant morts prématurément, c'est-à-dire avant d'avoir atteint l'âge de trente ans. Il reste donc trente-six officiers qui ont conclu au moins une alliance. Certains en ont contracté plusieurs : deux officiers se sont mariés deux fois et deux autres trois fois. En tout, nous comptons donc 41 mariages ${ }^{16}$.

12. Arch. dép. de Vendée, 1 E 683.

13. Les fausses déclarations d'ancêtres données par les magistrats poitevins, qui ne sont pas très nombreuses, avaient surtout pour but de masquer une lointaine origine marchande. Les officiers souhaitaient ainsi se démarquer du monde de la marchandise et du négoce, très important à Nantes.

14. Les ancêtres du maître Boutillier étaient apothicaires ou chirurgiens en même temps qu'ils régissaient les terres du seigneur de Beaupréau avant d'en contrôler les francs-fiefs et d'en devenir finalement les juges.

15. C'est le cas des familles Pichard, Robert ou encore Jannet qui cumulèrent le plus souvent des offices de judicature et de finance.

16. Nous avons ici déduit le mariage de l'auditeur François Soulard, sieur de la Roche, car nous ne connaissons pas la date de son mariage. 
Tableau 3 - Milieu social d'origine des beaux-pères des officiers

\begin{tabular}{|l|c|}
\hline \multicolumn{1}{|c|}{ Milieu social d'origine } & Part en \% \\
\hline Chambre des comptes & 7 \\
Officiers de finance & 18 \\
Officiers de justice & 25 \\
Officiers ministériels & 7 \\
Négociants, marchands & 11 \\
Officiers de maison princière & 4 \\
Militaires & 21 \\
Indécis & 7 \\
\hline \multicolumn{2}{|c|}{ Total } \\
\hline
\end{tabular}

Ces pourcentages, les professions des beaux-pères étant connues pour $68 \%$ d'entre eux, montrent qu'une bonne part de magistrats prend femme dans le monde judiciaire et financier. En effet, au moins $25 \%$ des fonctions exercées appartiennent à la robe, essentiellement petite ou moyenne, qu'elle soit royale ou seigneuriale. Deux officiers seulement ont épousé une fille de magistrat des comptes. L'alliance avec des familles militaires était également recherchée puisqu'elle concerne $21 \%$ des magistrats. Ces fonctions se déclinent du garde de la porte du roi au commandant des grenadiers de France.

On trouve enfin pour $11 \%$ des cas les alliances avec le monde du commerce et du négoce. Celui-ci est tout aussi divers que les fonctions militaires puisqu'il va du métier corporatif de marchand de draps de soie aux plus importantes familles négociantes nantaises. Les alliances conclues sont conformes à la hiérarchie interne de la Chambre : les correcteurs, les auditeurs et les greffiers en chef prennent leurs épouses principalement dans des milieux sociaux comparables, c'est-à-dire plutôt de moyenne bourgeoisie, alors que les maîtres et les présidents s'unissent principalement avec des familles de la noblesse et de la moyenne, voire de la haute, bourgeoisie.

\section{Une majorité de roturiers}

La Chambre des comptes représente pour les élites poitevines une opportunité d'ascension rapide vers la réussite en autorisant un anoblissement en deux générations successives.

20 \%, c'est-à-dire huit magistrats, étaient déjà nobles lors de leur entrée à la Chambre des comptes; celle-ci permettait un affermissement de leur noblesse récente souvent jugée légère. En l'occurrence, deux familles ${ }^{17}$ avaient acquis leur noblesse par l'achat de lettres à la fin du règne de

17. C'est le cas des familles Cicoteau et Panou. 
Louis XIV. Pour la moitié, les magistrats (soit vingt) étaient roturiers lors de leur entrée. Même si 30 \% des magistrats étaient en voie d'anoblissement ${ }^{18} \mathrm{ou}$, en d'autres termes, succédaient à leur père, il y eut seulement quatre familles anoblies avant 1790 sur les seize entrées roturières depuis le début du XvIII siècle. Les morts prématurées et la Révolution en sont les principales causes.

Ces chiffres ne doivent être pris que comme des estimations : comme le remarque Jean Meyer, la limite juridique qui sépare la noblesse de la roture est théoriquement très nette, mais en réalité il existe une assez large part d'indétermination qui frôle la noblesse d'usurpation ${ }^{19}$. Ainsi la famille Babin que l'on a comptée parmi les familles roturières vit ses armes enregistrées à l'Armorial Général en 1696 sans que l'on ait la preuve qu'elle ait acheté des lettres de noblesse. De même, parmi les deux familles qui prétendaient être nobles depuis le xvl $\mathrm{x}^{\mathrm{e}}$ siècle, l'une, les Voyneau du Plessis, eut un itinéraire singulier. Jeune orphelin, René Louis Voyneau, né en 1678 et héritier de ses parents décédés, fut élevé par un tuteur qui dut verser les francs-fiefs en son nom. S'il cessa de les payer tout le temps qu'il fut pourvu de l'office de commissaire général des milices de la capitainerie des Sables-d'Olonne, il dut se soumettre à nouveau à cette obligation quand il en fut dessaisi en 1716 au motif que son tuteur les avait payés pour lui pendant sa minorité. L'acquisition d'une charge d'auditeur des comptes ${ }^{20}$ en 1719 lui permit d'échapper définitivement à cette taxe. Pour dissiper toute contestation éventuelle sur l'appartenance de sa famille au second ordre, son fils aîné Louis Charles Édouard Voyneau, lui succéda dans son office d'auditeur en $1741^{21}$, afin de remplir les conditions de l'anoblissement graduel.

Grâce à leur nouvelle dignité, les officiers du grand Bureau, c'est-à-dire les présidents et les maîtres, s'attribuaient le titre de " chevalier " alors que les officiers moins bien placés dans la hiérarchie (auditeur, correcteur et greffier en chef) ne prenaient que celui d'" écuyer ». Le président de Chassenon, né dans la roture, se permettait ainsi de prendre le nom de " chevalier de Chassenon " et parfois la qualification de " Haut et Puissant seigneur de Chassenon ", surtout après son mariage en 1771 avec la fille du comte de Lanjamet. Néanmoins, les magistrats subalternes ne s'embarrassaient pas pour se parer eux aussi de tels titres.

18. C'est le cas de l'auditeur Louis-Charles Arnault de la Motte, de l'auditeur JeanFrançois-Aimé Cardin, du maître Nicolas-Joseph Rocquand de Pontbureau ou encore de l'auditeur Jean-Jude Badereau de la Caffinière.

19. MEYER, Jean, La Noblesse bretonne au XVIII siècle, Paris, 1966, tome 1, p. 197.

20. René-Louis Voyneau de Plessis descendait de René II Voyneau, son arrière-grandpère, qui avait épousé à Nantes en 1579 Claude Le Bloay, la fille de François Le Bloay, seigneur de Gargoule, et second président en la Chambre des comptes de Bretagne de 1565 à 1571.

21. PerocheAU, Joël, " À propos des Lettres de noblesse de relief de Noblesse des Sieurs Voyneau ", dans OLONA - Histoire des Sables-d'Olonne et de sa région, 1983, $3^{\mathrm{e}}$ trimestre, $\mathrm{n}^{\circ} 105$, p. 9-12; la crainte que ces paiements ne leur soient imputés à dérogeance poussèrent les enfants de René Louis Voyneau à adresser en 1771 des lettres de relief de noblesse à Louis XV qui statua et maintînt la famille en sa qualité noble. 
En règle générale, tous les magistrats se faisaient une haute opinion d'eux-mêmes, en particulier le maître Panou de Faymoreau dont la famille était noble déjà depuis trois générations. D'après son fils,

"[Il avait un] caractère orgueilleux, fier de sa noblesse, dédaignant tout ce qui était roturier. Il était généralement plus obéi qu'aimé, mais en revanche, $\mathrm{Mr}$ Faymoreau avait beaucoup d'esprit et d'imagination : il versifiait avec beaucoup de facilité, d'humeur gaie et à la Juvénal, jouait bien la comédie et brillait en société par ses jovialités et ses facéties à la Debureau, toujours accompagné d'un esprit caustique. C'était l'âme de toutes les parties de plaisir. Il chantait très bien la petite chansonnette en s'accompagnant de son violon, dansant bien le menuet, aimant surtout la table, libertin à l'excès, vrai Céladon volage et inconstant, changeant de maîtresse comme de chemise, tel était Mr de Faymoreau quand il songea à se marier ${ }^{22}$."

Les mœurs de ce personnage hautain et libertin sont à rapprocher de celles des officiers du parlement de Paris qui aimaient briller en société et appréciaient notamment la musique et les plaisirs de la table.

Les prétentions des magistrats récemment anoblis suscitaient parfois des tensions ${ }^{23}$. À ce sujet, les officiers subalternes ont dû subir des moqueries sur leur pseudo-noblesse, tel l'affront public qu'endura l'auditeur Armand Gabriel François Badereau, fils d'un auditeur et petit-fils d'un maître de poste. Des détracteurs jaloux auraient en effet payé des acteurs pour chanter devant lui au théâtre de Nantes : "Salut M. Badereau qui pour armes à trois zéros ". Ces " zéros " auraient eu comme principal symbolisme de rappeler la forme ordinaire du crottin de cheval!

\section{Les chemins de la Chambre des comptes}

Il convient à présent de voir si ces magistrats ont suivi les mêmes parcours scolaire et professionnel avant d'intégrer la Chambre des comptes de Bretagne.

\section{Des études à l'exercice d'une profession}

Les parcours scolaires sont à peu de chose près les mêmes : collège et université. Certains de ces officiers ont seulement accompli les trois ou

\footnotetext{
22. Extrait des Souvenirs inédits de Piter Panou de Faymoreau, collection privée.

23. Les descendants des Poitevins Jacques Jaudonnet, père et fils, auditeurs en la Chambre des comptes de Bretagne sous le règne de Louis XIV, avaient eux aussi été l'objet de bien des jalousies et de moqueries. Jacques-Antoine Jaudonnet, chevalier de LavauRicher, fils et petit-fils des précédents, eut beaucoup de mal à s'imposer sur sa propre terre tant son origine nobiliaire était fortement contestée par les autres gentilshommes de la paroisse, en particulier par Jacques Eleonor Bodet. Jaudonnet se plaignait en effet " a lesgard de ce quun certain quidant [Bodet] lequel est a la teste de deux ou trois cens mannans demande que jaye a leurs produire mes titres de noblesse, je suis pres de le faire devant le roy ou gens commis de sa part, et non pas devant un nombre de canaille, mal a propos mutiné contre moy. ", cité par PAINEAU, Pascal, Une paroisse du bocage Bressuirais au XVII siècle : Chanteloup, Mémoire de maîtrise, université de Poitiers, 1982, p. 61.
} 
quatre premières années du collège avant d'entrer chez un notaire ou un procureur postulant pour y devenir clerc $^{24}$.

Le plus souvent, ces futurs magistrats ont fréquenté les collèges baspoitevins de Luçon et de Fontenay-le-Comte. Ceux qui étaient originaires du nord de la région se sont, quant à eux, orientés vers les écoles ou les collèges de Nantes : l'un des frères cadets du maître Boutillier décéda ainsi à la fin de l'année 1780 chez les frères des Écoles Chrétiennes de Saint-Similien à Nantes ${ }^{25}$. Il arrivait que le collégien, pour terminer sa formation, fût envoyé dans la capitale, comme ce fut le cas pour le futur maître Jacques Louis Panou de Faymoreau. Dans une lettre datée du début de l'année 1767, sa mère évoquait l'envoi à Paris de son fils :

"Mon fils [alors âgé de quinze ans] a passés les faistes avec nous avec un père loratoir qui est son régent et fort aimable, il na plus que six mois pour finir ses classe dont il est bien contant; nous contons lanvoyer a Paris [pendant] ses vacance, ce sejour luy plaisra plus que celuy du college ${ }^{26}$."

Malgré tout, Pierre, le fils cadet du seigneur de Faymoreau, faisait peu d'éloges sur l'éducation de son père et d'un ton acerbe notait que

"l'éducation de Mr de Faymoreau a été très négligée : elle se ressentait beaucoup de l'époque et du temps où la noblesse se distinguait plûtot par les armes que par l'instruction : il ne mettait pas un mot d'orthographe et écrivait souvent mal sa langue ainsi qu'on peut en juger par l'enterrinement qu'il fit au testament olographe de son père et de tous les autres écrits et lettres que j'ai de lui. Dénué de toute érudition en histoire, en géographie, sachant à peine faire ses quatres règles, il n'avait aucune connaissance dans les sciences ${ }^{27}$."

Après le collège, au moins la moitié de ces Poitevins ont réalisé un cycle de droit en trois ans dans une université, en l'occurrence à Poitiers, pour obtenir le grade de licencié ès lois. Ils sont sans doute plus nombreux mais l'absence de documents ne permet pas d'être plus précis. Près d'un tiers de ces licenciés a, après avoir quitté l'université, prêté, la main sur les Évangiles, le serment d'avocat; ils ont donc suivi un stage de deux ans puis se sont inscrits sur le tableau comme avocat plaidant. Ainsi Jean-Louis Biaille, âgé de vingt-deux à vingt-trois ans, passa l'année 1756 à Paris occupé à suivre les audiences puis s'installa à Nantes pendant une partie de l'année 1757 vraisemblablement pour la même raison. Tous sont ultérieurement dits " avocat en parlement " à l'exception de quatre qui le sont au " parlement de Paris ». Seul Simon Bourdeau, semble-t-il, s'était tourné vers la fonction de procureur après l'université, puisqu'on le retrouve à l'âge de 23 ans chez maître Ferrat le Jeune, procureur au parlement de Paris ${ }^{28}$.

24. Ce fut le cas de Jean Biaille qui " dès sa première jeunesse [...] était clerc chez $M$. Mauflâtre Procureur. " dans Arch. dép. de Vendée, 1 E 683.

25. Arch. dép. de Loire-Atlantique, $25 \mathrm{~J}$.

26. Arch. dép. de Loire-Atlantique, 2 E 735.

27. Extrait des Souvenirs inédits de Piter Panou de Faymoreau, collection privée.

28. Arch. dép. de Vendée, 1 E 681. Maître Ferrat le Jeune demeurait alors rue Galande, paroisse de Saint-Séverin. 
Quatre officiers (10\%) ont été tentés par une carrière médicale. C'est le cas, entre autres, du futur greffier en chef Jean-Baptiste Alexis Pichard qui fut agrégé de médecine au collège royal de La Rochelle ou bien encore du président François Gabriel Duval de la Vergne ou de l'auditeur Charles Lezin Cossin, tous deux docteurs de la faculté de médecine de Montpellier.

Si l'on en juge par l'échantillon étudié, les parcours scolaires les ont peu portés à fréquenter des institutions scolaires étrangères à leur province. La présence d'une faculté de droit à Poitiers, de bonne réputation quoique déclinante, dispensait d'aller étudier ailleurs, même s'il était encore de bon ton à l'époque de quitter sa province natale et de fréquenter plusieurs institutions scolaires, sous peine de voir ses compétences mises en doute ${ }^{29}$.

Quatorze officiers (35\%) n'ont pratiqué aucune profession avant d'intégrer la Chambre et les deux tiers d'entre eux étaient des fils de conseillers déjà présents dans la compagnie. La Chambre se montrait donc très accueillante pour les enfants de ses officiers et pour ceux qui étaient d'origine noble. En effet, Jacques Louis Panou et Louis Venant Cicoteau n'avaient pas vingt ans quand ils furent établis dans leur office. Sept officiers (17,5\%) étaient avocats (dans une sénéchaussée, un présidial ou au parlement de Paris). Trois autres (7,5\%) avaient auparavant exercé une fonction militaire comme Nicolas Joseph Rocquand de Pontbureau qui avait été commissaire aux chasses de Saint-Gilles-sur-Vie. Ces charges militaires n'étaient que des fonctions subalternes qui permettaient de rester près de chez soi. Sept officiers $(17,5 \%)$ ont détenu des offices de finances, principalement dans les élections de Fontenay et de Châtillon comme Louis Charles Édouard Voyneau du Plessis ou Jacques Panou de Faymoreau qui cumulèrent tous deux un office de conseiller en l'élection de Fontenay-Le-Comte. Cinq magistrats $(12,5 \%)$ ont exercé un office de justice dans une juridiction royale ou seigneuriale, comme Marin Jean-Baptiste Boutillier de la Chèze qui était juge de la châtellenie de Mortagne. Deux officiers (5\%) ont hésité entre offices de judicature et de finance. Marie Augustin Pichard de la Caillère fut conseiller en l'élection de Fontenay puis juge au siège royal de cette même ville alors que Pierre Jacques Robert des Essertons avait été receveur des décimes avant de devenir sénéchal de la principauté de Talmont. Enfin, deux magistrats aux comptes (5\%) étaient médecins mais ils occupèrent en même temps un office de judicature ou de finances. Deux Poitevins, enfin, furent reçus à plus de 60 ans au terme d'une vie professionnelle déjà bien remplie ${ }^{30 .}$

29. CASSAN, Michel, «Formation, savoirs et identité des officiers "moyens" ", dans CASSAN, Michel (dir.), Les officiers " moyens " à l'époque moderne, Limoges, 1998, p. 302.

30. François Gabriel Duval de la Vergne, président en l'élection de Fontenay de 1722 à 1759, reçu président à l'âge de 67 ans en 1759; Jean Cardin, alors assesseur au siège royal de Fontenay-le-Comte, obtient un office anoblissant d'avocat secrétaire ordinaire du roi de Pologne Stanislas, fonction tout à fait honorifique. En 1764, il n'est plus assesseur mais 
Lors de leur réception, certains magistrats durent résigner leur office, bon gré mal gré. En 1781, le médecin Jean-Baptiste Alexis Pichard déclarait :

"Que comme il exerçoit cy devant la profession de médecin qui est incompatible avec les fonctions de l'office de greffier en chef de la Chambre des Comptes de Bretagne dans lequel il a l'honneur de solliciter sa reception, prévoyant que cette incompatibilité pouroit être un obstacle a sa reception, il requiert à ces causes qu'il vous plaises, nos seigneurs luy donner acte de la déclaration qu'il fait de renoncer à l'exercice de la profession de médecin pour ne s'occuper que des fonctions qui regardent l'office de greffier en ladite cour ${ }^{31}$."

La Chambre n'autorisait donc pas que ses officiers « docteurs en medecine " continuassent d'exercer leur fonction, la jugeant incompatible avec un emploi dans une cour souveraine.

Pour d'autres, l'abandon de la charge exercée s'expliquait par des raisons d'ordre politique. François Soulard, lieutenant en l'élection de Châtillon-sur-Sèvre avant sa réception en 1786 dans un office d'auditeur, était aussi un des subdélégués de l'intendant de Poitiers " mais craignant que les fonctions de subdélégué de l'intendant de Poitiers qu'il exerce aux fins de commission, soient regardées incompatibles avec l'office de conseiller auditeur en cette cour, il déclare se désister a peu et a plein de ladite commission de subdélégué et renonce des a présent a en faire a l'avenir aucunes fonctions; voulant que les pouvoirs qu'il en a reçu, soient comme non avenues ${ }^{32}$ ». Une Chambre des comptes de province, soucieuse de son indépendance, voyait en effet d'un mauvais œil qu'un de ses magistrats soit lié à un agent du pouvoir royal.

Tous les officiers n'ont cependant pas résilié leurs fonctions avant leur entrée à la Chambre. Quatre officiers ont conservé leur charge et quatre autres ont été reçus dans un autre office alors qu'ils étaient titulaires d'un office aux comptes ${ }^{33}$. Joseph Rocquand, correcteur depuis 1746, était aussi lieutenant général de la capitainerie garde-côtes de Beauvoir-sur-Mer et commissaire aux chasses de Saint-Gilles-sur-Vie. De cette dernière fonction, il déclarait le 7 février 1749 au greffe de la Chambre des comptes qu'il avait " remercié depuis quelques temps au Ministre de la Marine la commission qu'il tenoit de lui pour faire les fonctions de commis aux chasses dans la port de Saint-Gilles du Poictou et qu'il renonce à les faire à l'avenir ${ }^{34}$ ". Il fit passer cette charge à son fils Nicolas Joseph Rocquand alors que ce dernier se faisait recevoir dans un office de conseiller maître. L'auditeur Charles

devient l'année suivante échevin de la capitale bas-poitevine. C'est à l'âge de 60 ans qu'il est reçu greffier en chef.

31. Arch. dép. de Loire-Atlantique, B 526.

32. Arch. dép. de Loire-Atlantique, B 535.

33. La raison semble être que l'office possédé avant ou acheté pendant l'exercice de leur office à la Chambre est estimable et honorable et ne déroge pas à l'exercice d'un office dans la seconde cour souveraine.

34. Arch. dép. de Loire-Atlantique, B 481. 
Laurent Bouhier devint maire des Sables-d'Olonne en 1751, seulement une année après sa réception dans un office d'auditeur. Quant à l'auditeur Pierre Jacques Robert et au greffier en chef Jean-Louis Biaille, ils furent tous deux reçus sénéchal de la principauté de Talmont quelques mois seulement avant leur accès à la Chambre et ils gardèrent cette fonction de juge pendant l'exercice de leur office. La raison de ce double cumul semble être que l'office supplémentaire, estimable et honorable, ne dérogeait pas à l'exercice d'un office dans une cour souveraine.

\section{L'importance de disposer d'appuis}

Acquérir un office à la Chambre ne se faisait pas sans aide. En plus de la solidarité familiale, il pouvait être utile de bénéficier de la protection de personnages puissants tels que les intendants de province ou les grands nobles. Ces magistrats ne dérogent pas à la règle puisque nous les retrouvons liés, eux et leurs familles, à des intendants et à de grands seigneurs. L'auditeur François Soulard avait été, avant son entrée à la Chambre des comptes, le subdélégué de l'intendant du Poitou à Châtillon, c'est-à-dire son homme de confiance. D'autres magistrats poitevins se sont alliés à des familles au service d'intendants. En tout, au moins six d'entre eux eurent des liens directs avec les intendants de Tours et du Poitou. Jacques Louis Bourasseau, le père de Jacques Joseph Marie reçu auditeur en 1779, après s'être marié à Maulévrier avec Catherine Jeanne Poupard, était devenu subdélégué de l'intendant de la généralité de Tours à Maulévrier. Et un de ses successeurs en 1761 fut Henri Élie Cossin, le frère du médecin Charles Lezin Cossin qui fut reçu auditeur à la Chambre en 1779. Alors qu'il n'était pas greffier en chef, Louis Marie Arnault, quant à lui, épousa la fille du sénéchal et du subdélégué de l'intendant du Poitou à La Châtaigneraie et fut reçu cinq mois après dans la cour bretonne.

La moitié au moins des magistrats poitevins eurent des liens concrets avec des gens de la haute noblesse, soit que leurs ancêtres ou eux-mêmes fussent employés à leur service ou qu'ils entrassent simplement en relations occasionnelles avec eux, leur rendant tel ou tel service ponctuel. Ainsi, les ancêtres du maître Marin Jean-Baptiste Boutillier de la Chèze avaient eu la responsabilité de la recette des terres des seigneurs de Beaupréau et de Cholet présents à la cour, et son père avait géré celles du duc de Neufville de Villeroy, baron de Mortagne. Jude Badereau, alors qu'il n'était pas encore auditeur, était au service du seigneur de Montaigu, Turpin de Crissé, le marquis de Crux. Il plaça ainsi une partie de sa nombreuse progéniture sous la protection de ce grand seigneur. Et lorsque Jude Badereau fut reçu auditeur en 1724, deux membres de la famille Turpin de Crissé étaient puissamment établis en Bretagne puisque l'un était évêque de Rennes et l'autre évêque de Nantes ${ }^{35}$. Sa belle-famille disposait également de relations solides puisque son beau-père, Jean de Courcelles, avait été le maître

35. MEYER, Jean, La noblesse bretonne, op. cit., p. 1132. 
d'hôtel de l'évêque de Luçon, Nicolas Colbert, le frère du ministre, puis celui du prince de Vermandois, le fils de Louis XIV et d'une de ses favorites Louise de la Vallière, qui vécut de 1667 à 1683. Nous pourrions multiplier les exemples.

Toutefois, le cas le plus intéressant concerne la famille des présidents Duval. Déjà, François Gabriel Duval de la Vergne avait, par sa position (il avait été pendant 37 ans président de l'élection de Fontenay-le-Comte), rendu service à plusieurs nobles de cour, ainsi qu'à de hauts magistrats parisiens et de grands ecclésiastiques qui avaient des intérêts en Bas-Poitou. Dans les années 1740 par exemple, il se disait dévoué à " Madame Françoise Gabrielle de Châteaubriant, marquise de la Savonnière, veuve de haut et puissant seigneur René Georges de Savonnière, marquis de Lignière ${ }^{36}$ ". Il défendait alors les intérêts que la marquise pouvait avoir dans la région et, en son nom, lui affermait des fermes et des logis ${ }^{37}$. En même temps, il s'occupait de ses affaires de succession ${ }^{38}$. Son fils, Duval de Chassenon, qui lui succéda dans son office de président à la Chambre des comptes en 1763, noua quant à lui des liens dès la fin des années 1760 dans les plus hautes sphères politiques de l'époque. Grâce à sa correspondance ${ }^{39}$, on apprend que lors d'un de ses séjours dans la capitale, du 21 mai au 13 juillet 1768, il résidait dans un des quartiers les plus chics, à l'hôtel de Turin, rue des Grands Augustins en la paroisse de Saint-André-des-Arts. Il côtoyait alors le premier président du parlement de Paris, le marquis d'Aligre ${ }^{40}$, qui était aussi comte de Marans (ville et port à la limite de l'Aunis et du Bas Poitou) et passait une partie de son temps en compagnie de l'intendant Sénac de Meilhan, le protégé de Choiseul ${ }^{41}$. Cette présence dans la capitale était donc tout à la fois l'occasion pour lui de montrer sa réussite et d'entretenir des relations précieuses qu'il pouvait faire fructifier ensuite en province.

\section{Des carrières classiques}

Si l'on s'intéresse maintenant à la carrière de ces officiers, on doit rappeler que pour entrer à la Chambre, le récipiendaire pouvait acheter une

36. Arch. dép. de Vendée, 3 E 36/398.

37. Arch. dép. de Vendée, 3 E 36/401.

38. Arch. dép. de Vendée, 3 E 36/358.

39. Arch. dép. de Vendée, 2 E 71.

40. Etienne François d'Aligre, comte de Marans, marquis d'Aligre (1727-1798), fut reçu conseiller au parlement de Paris en 1745, président à mortier en 1752 et enfin premier président de 1768 à 1771 et de 1774 à 1788. Comme le Président de Chassenon, le marquis d'Aligre est intéressé dans les marais desséchés du Bas-Poitou.

41. Gabriel Sénac de Meilhan (1736-1803), avocat au parlement de Paris en 1762, il obtient une charge de conseiller au grand conseil, émanation du conseil du roi, et entame une brillante carrière administrative, grâce à de solides appuis à la cour, notamment celui de Choiseul dont il fréquente la sœur, la comtesse de Tess. Il achète une charge de maître des requêtes au conseil d'État en 1763 et devient successivement intendant de la Guadeloupe en 1763, des îles de France et de Bourbon, de La Rochelle en 1766. En 1765, il obtient le privilège des " entrées dans la chambre du roi ". 
charge en ayant bénéficié de la résignation d'un officier et le traité d'office était entériné devant notaire. Le résignant devait toutefois, s'il voulait résigner à la personne de son choix, payer la dispense des quarante jours. L'office pouvait être accompagné des lettres de provisions ou de leurs copies précédentes. Ainsi, le 5 juin 1767, Louis Charles Édouard Voyneau s'obligeait de remettre à Jean Reliquet « incessament ses provisions et celles de ses prédecesseurs dans ledit office avec toutes les autres pieces et quittances de marc d'or au soutien ensemble la robe de ceremonie ${ }^{42}$ ". Mais le plus souvent, la vente se faisait à simple procure, c'est-à-dire que le résignataire devait aller directement se faire pourvoir à la chancellerie et payer ainsi tous les frais supplémentaires.

Une fois pourvu, le prétendant se présentait à la Chambre et entreprenait les démarches auprès du procureur général et du premier président pour se faire recevoir. Si le magistrat était apparenté à un ou plusieurs magistrats en place dans la compagnie, les démarches prenaient logiquement moins de temps. Le procureur général nommait alors deux maîtres qui s'occupaient d'auditionner quatre témoins pour "l'information de vie et de mœurs " et de prendre également sa déposition au sujet de sa généalogie pour vérifier s'il n'était pas un descendant d'un comptable resté redevable au roi. Une fois ces témoignages entendus, le récipiendaire devait passer un examen sur la pratique des finances. Généralement, cela concernait l'examen d'un compte ou la réformation d'un domaine. Puis on procédait à la réception. Le nouvel officier prêtait serment et offrait un banquet à l'ensemble de ses collègues ${ }^{43}$.

La correspondance entre Pierre Jacques Letard et son père, de Poitiers, nous livre des indications originales sur les pratiques et les moyens d'acquérir un office à la Chambre ${ }^{44}$. Issu d'une famille de bourgeoisie robine de Poitiers ${ }^{45}$, Pierre Jacques Letard épouse la fille aînée du correcteur JeanVictor Gabriel Chauvière de la Pagerie. Ce dernier, sans héritier, offre son office de correcteur en dot à son gendre, en plus de quelques domaines. La jeune mariée décède prématurément à la fin de l'année 1784, soit un an après le mariage; les relations entre le gendre et le beau-père se durcissent alors brutalement du fait du problème du paiement de la dot. Après bien des marchandages par l'intermédiaire de leurs avocats, un acte est finalement passé entre Letard et son beau-père, par lequel le gendre préfère acquérir l'office de son beau-père plutôt que les domaines. Le 25 février 1785, de Mortagne, il écrit à son père : « J'ai enfin terminé mes affaires avec Mr de la Pagerie, notre acte fut solennellement signé mercredi dernier, et

42. Arch. dép. de Loire-Atlantique, 4E 2/1068.

43. MEYER, Jean, La noblesse bretonne..., op. cit., p. 192.

44. Arch. dép. de la Vienne, J 142.

45. Son père Jacques Jean-Baptiste Letard était procureur à la cour des monnaies de Poitiers, son grand-père le fut également, alors que son bisaïeul et son trisaïeul étaient procureurs au présidial de la même ville. 
cimenté par une foule de caresses et de choses obligeantes qu'il me prodigua. Je n'assurerais pas que vous approuverés l'arrangement que j'ai fait; il n'est pas cepandant point contraire à mes interêts. Je me suis accomodé de la charge, $[\ldots]$ laquelle $[\ldots]$ joint à la necessité dans laquelle je me trouve d'avoir une place, ma fait preferer l'office de correcteur et ce qui l'accompagne, aux domaines que j'aurais eux [...]. » Dans la foulée de cet acte, Pierre Jacques Letard estime devoir aller à Nantes " pour mes affaires présentes " dans l'intention " de me faire recevoir dans la charge qui m'a été cédée". Il compte en effet s'entretenir avec " quelques officiers de la Chambre, pour savoir d'eux combien il leur en a coûté pour se faire recevoir ». On aurait pu penser que son beau-père l'aurait aidé dans la délivrance de ses provisions mais il semblerait qu'il lui ait juste expliqué les démarches à entreprendre. Toutefois, il a vraisemblablement dû lui dire ne pas solliciter " en cette occasion [le] ministere d'un procureur ". Cela éviterait selon lui " une branche de dépense qui réside ordinairement dans la personne du procureur que j'aurais emploié " car, paraît-il, il " rend ordinairement ce service aux recipiendaires moiennant bonne finance ". Son but est donc de demander à quelques officiers " un état exact des différens frais qu'il faudra faire " pour ensuite, de retour à Poitiers, écrire "à un secrétaire du roi, de m'obtenir des provisions". Mais dans une lettre envoyée le 3 mars de Nantes, Pierre Jacques Letard écrit à son père qu'il n'est plus très décidé à garder sa charge et qu'il souhaite la vendre avantageusement. Pour ce faire, il rapporte " que le semestre de mars est ouvert depuis lundi " et que " cette époque réunit ordinairement une très grande partie de la Chambre, les différentes personnes qui cherchent à s'y placer ne manque gueres de choisir la rentrée des officiers, dans l'idée qu'il s'en trouvera parmi eux qui voudront vendre " et il ajoute que "si j'avais le bonheur de rencontrés un envieux qui voulut payer l'avantage d'être nommé monseigneur, je l'aurais bien vite accomodé de ma dignité ».

Ces précisions montrent clairement qu'il est possible d'acquérir une charge pour une personne " qui cherche [...] à s'y placer " en allant directement à la Chambre s'entretenir avec des officiers désireux de vendre leur office. Et le meilleur moment pour cela est l'ouverture d'un semestre, en l'occurrence ici celui de mars qui s'ouvre le $1^{\text {er }}$ du mois (après dix jours d'interruption des services de la cour souveraine) réunissant ainsi, comme l'indique Pierre-Jacques Letard, " une très grande partie de la Chambre ". Pour le fin mot de l'histoire, notre homme se décida finalement à garder son office et se fit recevoir à la Chambre le 14 septembre 1785.

À quel âge ces magistrats ont-ils été reçus ? Parmi les quarante cas étudiés, vingt-deux intègrent la Chambre avant l'âge de 30 ans $^{46}$. Les Poitevins entrent donc jeunes. Après 30 ans, la rupture est brutale, les entrées se

46. Pour un seul magistrat (l'auditeur François Soulard) nous ne connaissons pas la date de naissance. Duval de Chassenon fut maître puis président, il est donc compté deux fois. 
font plus rares jusqu'à la barre des 50 ans. Au-delà on remarque un léger redressement du nombre d'entrées, puisque dix magistrats ont acquis un office après 50 ans, ce qui laisse transparaître l'achat d'un office en fin de carrière. On peut donc distinguer deux attitudes principales : celle des officiers qui commencent leur vie professionnelle dans la Chambre et celle des magistrats qui la terminent.

Tableau 4 - Âge des officiers poitevins à leur entrée en fonction

\begin{tabular}{|c|c|c|c|c|c|c|}
\hline Âge & Président & Maître & Correcteur & Auditeur & Greffier en chef & Total \\
\hline $18-24$ & & 2 & & 5 & & 7 \\
$25-29$ & 1 & 3 & & 5 & 2 & 11 \\
$30-34$ & & & 2 & 2 & & 4 \\
$35-39$ & & 1 & & 3 & & 4 \\
$40-44$ & & & & 3 & 1 & 4 \\
$45-49$ & & & & 2 & 2 & 4 \\
50 et + & 1 & & 1 & 3 & 1 & 6 \\
\hline Total & 2 & 6 & 3 & 23 & 6 & 40 \\
\hline
\end{tabular}

Les offices de maître et d'auditeur ont été achetés en priorité par des Poitevins jeunes, cinq avant 30 ans pour les six offices de maitre et dix pour les vingt-trois offices d'auditeur. Seuls six offices, dont trois d'auditeur, ont été achetés à la fin d'une ascension sociale. Quant aux trois correcteurs, ils entrent dans la Chambre à un âge plutôt tardif, avec une carrière déjà bien engagée, voire sur la fin. Six officiers entrés après 45 ans ont un fils qui leur succède dans la décennie suivante. La famille peut ainsi bénéficier des effets de l'anoblissement graduel en l'espace de 25 à 30 ans.

Seuls seize magistrats (40\%) ont pu accomplir au minimum vingt ans d'exercice avant que la mort ne les frappe ou que la Révolution ne les surprenne. Douze sont morts titulaires sans avoir fait vingt ans et douze autres ont perdu leur office avec la Révolution, alors que trois d'entre eux avaient dépassé vingt ans de service comme le greffier en chef Jean Cardin ou le maître Jacques Louis Panou de Faymoreau. Le déclenchement soudain de la Révolution leur a interdit d'acquérir des lettres d'honneur.

Tableau 5 - Durées d'exercice des officiers poitevins

\begin{tabular}{|l|c|c|c|c|c|c|}
\hline & $\begin{array}{c}\text { Morts } \\
\text { titulaires }\end{array}$ & $\begin{array}{c}\text { Résignation } \\
\text { entre } 20 \text { et } \\
21 \text { ans }\end{array}$ & $\begin{array}{c}\text { Résignation } \\
\text { entre } 21 \text { et } \\
25 \text { ans }\end{array}$ & $\begin{array}{c}\text { Résignation } \\
\text { après } 25 \text { ans }\end{array}$ & $\begin{array}{c}\text { Suppression } \\
\text { par la } \\
\text { Révolution }\end{array}$ & $\begin{array}{c}\text { Cas } \\
\text { particulier }\end{array}$ \\
\hline Nombre & 12 & 5 & 6 & 5 & 11 & 1 \\
\hline
\end{tabular}


Si l'on calcule la durée d'exercice des officiers, on trouve vingt-cinq ans de service en moyenne. C'est nettement moins que la moyenne de trente et un ans des auditeurs avancée par Guy Saupin ${ }^{47}$ pour la période de 1772 à 1790, ce qui signifie que les officiers poitevins ont moins tardé que les autres à revendre leurs charges une fois les conditions juridiques remplies. Douze sont morts en charge, soit plus d'un quart d'entre eux. C'est beaucoup mais logique si l'on considère que les magistrats ont acquis leur charge à la fin de leur carrière ${ }^{48}$. Le cas particulier concerne Jean-Louis Biaille de Lengibaudière qui fut condamné par le parlement de Paris à être déchu de ses fonctions de greffier en chef et de sénéchal après avoir été condamné au bagne.

Cinq magistrats ont résigné leur charge après l'avoir exercée plus de vingt-cinq années, comme Paul-François Babin, sieur des Ardilliers, qui a gardé son office pendant un temps exceptionnel de plus de trente-huit années. Quant à Pierre I Rochard, âgé de 47 ans seulement, il résigne son office d'auditeur qu'il avait exercé vingt-quatre ans « son âge ne lui permettant plus d'en continuer les fonctions ${ }^{49} "$ !

\section{Un office aux comptes : un investissement rentable}

Au-delà de l'honneur que confère un office aux comptes, quel en est l'impact sur la fortune des magistrats?

\section{Le coût des offices}

Si l'on se fie aux vingt-sept traités d'office retrouvés, les offices acquis par les Poitevins se situent dans une fourchette de prix comprise entre 16000 livres pour une charge de greffier en chef et 54000 livres pour celle de président ${ }^{50}$.

Nous remarquons qu'entre 1730 et 1770 , les prix des offices sont largement sous-estimés. Les charges de président tournent en général autour de 70000 livres ${ }^{51}$ alors qu'ici elle est estimée 54000 livres. Faut-il y voir un rééquilibrage tarifaire ou simplement un camouflage des prix réels? Les actes notariés ne montrent effectivement pas les dessous-de-table qui sont bien plus nombreux qu'on ne le pense. Louis-Charles-Edouard Voyneau, seigneur du Plessis-Mauclerc, écrivait le jour de l'Ascension 1767 à un de ses cousins, à propos de la vente de son office d'auditeur : « Je pars demain pour

47. SAUPIN, Guy, Les origines sociales des conseillers à la Chambre des comptes de Nantes (1772-1790), étude des familles, mémoire de maîtrise, université de Nantes, p. 30.

48. Il y eut toutefois trois décès prématurés : l'auditeur Jean-Jude Badereau décédé à l'âge de 29 ans, l'auditeur Louis-Venant Cicoteau à l'âge de 32 ans et son frère PierreThomas Cicoteau à l'âge de 22 ans.

49. Arch. dép. de Loire-Atlantique, B 100.

50. Arch. dép. de Loire-Atlantique, 4 E 2/236. Cet office de président acheté en 1759 54000 livres est revendu en 1781 au prix de 76500 livres.

51. MeYER, Jean, La Noblesse bretonne..., op. cit., p. 181. 
Tableau 6 - Prix des offices achetés et vendus par les officiers poitevins (en livres tournois)

\begin{tabular}{|c|c|c|c|c|c|}
\hline & Président & Maître & Correcteur & Auditeur & $\begin{array}{c}\text { Greffier en } \\
\text { chef }\end{array}$ \\
\hline 1716 & & & & 33000 & \\
\hline 1721 & & & & 39500 & \\
\hline 1724 & & & & 37500 & \\
\hline 1729 & & & & 26000 & \\
\hline 1733 & & 50000 & & & \\
\hline 1736 & & & & 26000 & \\
\hline 1736 & & 49000 & & & \\
\hline 1744 & & & & 26000 & \\
\hline 1746 & & & 24000 & & \\
\hline 1748 & & & & 26000 & \\
\hline 1749 & & & & 26000 & \\
\hline 1749 & & & & & 16000 \\
\hline 1750 & & & & 26000 & \\
\hline 1758 & & 50000 & & & \\
\hline 1759 & 54000 & & & & \\
\hline 1762 & & & 33000 & & \\
\hline 1763 & & 50000 & & & \\
\hline 1767 & & & & 26000 & \\
\hline 1769 & & & & 26000 & \\
\hline 1775 & & & & 40000 & \\
\hline 1778 & & & & 37000 & \\
\hline 1778 & & & & 39000 & \\
\hline 1779 & & & & 40000 & \\
\hline 1781 & 76500 & & & & \\
\hline 1783 & & & 31000 & & \\
\hline 1784 & & & & 36000 & \\
\hline
\end{tabular}

Nantes, ayant reçu une lettre de l'Epertière ${ }^{52}$ qui offre 44000 lt et 25 louïs de pot de vin, avec les mêmes conditions que je luy proposois excepté que les deux payements de 12000 livres et de 6000 livres se feront à Nantes, tous les autres à Fontenay. Je luy ai mandé que je le verrais incessamment et que j'en ferois plus dans un entretien d'une demie heure que par vingt lettres ${ }^{53}$. " Ces chiffres sont forts différents de ceux du contrat passé devant notaires le 5 juin suivant, soit deux semaines plus tard, qui stipule que la

52. Jean-René Reliquet de l'Epertière.

53. Arch. dép. de Loire-Atlantique, 1 E 323. 
vente se fait moyennant un prix de 26000 livres à payer en huit payements de 3000 livres et un de 2000 en la ville de Fontenay-le-Comte! Où sont donc passées les 18000 livres qui manquent entre la lettre et le contrat? Ce n'est tout de même pas rien, c'est le prix d'un office de greffier en chef! Voyneau avait-il conclu avec son acquéreur un accord sous seing-privé " avant notaire " dans lequel étaient soigneusement stipulées les clauses de la vente? S'il n'y a pas à douter que les dessous-de-table existent, un tel écart de prix fausse tous les traités d'office passés devant notaires. Le prix de 44000 livres n'est pas exorbitant en soi puisque certains offices d'auditeur se négocient jusqu'à 40000 livres, voire un peu plus ${ }^{54}$. Jean Meyer indique également que les chiffres notariaux sont des chiffres de façade mais que l'écart entre le prix notarial et les prix réels est très faible. Ici, ce n'est pas le cas. Avec un seul exemple, il n'est pas possible de généraliser mais nous pouvons dire que les chiffres notariaux restent tout de même en dessous de la réalité et sont donc à manipuler avec précaution.

Le mariage, avec la dot, constitue un moyen pour financer l'achat d'un office. Si l'on compare les dates de mariage et celles de l'entrée en charge, on constate que treize conseillers ont pris femme avant d'intégrer la compagnie et douze après. Pour dix d'entre eux, les dates sont très proches. Il apparaît alors que le mariage est conclu en prévision de l'achat de l'office, la dot de l'épouse servant ainsi à son financement. Cela peut toutefois être aussi le cas des mariages célébrés quelques années après l'entrée à la Chambre des comptes. Jacques Joseph Jannet, qui avait acquis un office d'auditeur en 1737, épousa en 1745 Louise Barthélémy, la fille d'un négociant nantais, et reçut de sa belle-famille 40000 livres dont « lesdits deniers observant et s'obligeant le futur de les employer au payement de ce qu'il doit de sa charge d'auditeur affain qu'elle soit subrogée aux droits du créancier et ait son hypothèque spéciale et privilégiée sur elle ${ }^{55}$ ".

Sur les vingt-trois contrats de mariage que nous avons trouvés (soit un peu plus de la moitié sur les quarante-trois possibles), quatre ne donnent pas de renseignements sur le montant des dots, soit que l'on désire cacher une fortune importante, soit au contraire que l'on préfère taire une fortune médiocre ${ }^{56}$. Sur ces quatre contrats, il s'en trouve trois d'auditeurs et un de greffier en chef. À première vue, ces offices modestes laissent supposer une fortune peu considérable ${ }^{57}$. Sur les dix-neuf contrats exploitables, $37 \%$ donnent le seul chiffre de l'apport féminin à l'image du mariage BourdeauBouron en 1741, alors que $42 \%$ donnent les deux montants dotaux. Comme

54. MEYER, Jean, La Noblesse bretonne..., op. cit., p. 182.

55. Arch. dép. de Loire-Atlantique, 4 E 2/361, Boufflet not. à Nantes, 21 février 1745.

56. MEYER, Jean, La Noblesse bretonne..., op. cit., p. 201.

57. De ces vingt-trois contrats, deux sont imprécis car nous ne connaissons que la somme des deux dots réunies (Pierre I Rochard et Renée Marguerite Courtin apportent "plus de 10000 livres à eux deux ", et Marie-Augustin Pichard et Marie-Anne Quéré apportent tous deux 37200 livres. Arch. dép. de Vendée, 2 C 119 et 2 C 307) et un autre passé en 1792 sort du contexte du financement d'un office. 
le montrent les quelques exemples ci-dessous, les apports des femmes sont généralement supérieurs à ceux des hommes.

Tableau 7 - Quelques exemples de dots selon les catégories d'officiers

\begin{tabular}{|c|c|c|c|c|c|}
\hline Date & \multicolumn{2}{|c|}{ Noms } & Dots (en livres) & Office \\
\hline 29-05-1741 & Bourdeau Simon & $\begin{array}{c}\text { Bouron Marie- } \\
\text { Claire }\end{array}$ & - & 8000 & Greffier en chef \\
\hline $04-10-1762$ & $\begin{array}{c}\text { Pichard Jean- } \\
\text { Baptiste A. }\end{array}$ & $\begin{array}{c}\text { Masson Marthe } \\
\text { Suzanne }\end{array}$ & 16000 & 18000 & Greffier en chef \\
\hline $21-02-1745$ & $\begin{array}{c}\text { Jannet Jacques } \\
\text { J. }\end{array}$ & $\begin{array}{c}\text { Barthélémy } \\
\text { Louise }\end{array}$ & - & 40000 & Auditeur \\
\hline $10-04-1745$ & $\begin{array}{c}\text { Babin Paul } \\
\text { François }\end{array}$ & $\begin{array}{c}\text { Fagondo Marie- } \\
\text { Julie }\end{array}$ & 33000 & 47000 & Auditeur \\
\hline $30-11-1783$ & $\begin{array}{c}\text { Letard Pierre } \\
\text { Jacques }\end{array}$ & $\begin{array}{c}\text { Chauvière } \\
\text { Marie-Victoire }\end{array}$ & 20000 & 31000 & Correcteur \\
\hline $22-04-1773$ & $\begin{array}{c}\text { Panou Jacques } \\
\text { L. }\end{array}$ & $\begin{array}{c}\text { Deurbroucq } \\
\text { Marie-Adr. }\end{array}$ & 12500 & 80000 & Maître \\
\hline $02-05-1771$ & $\begin{array}{c}\text { Duval Franç } \\
\text { Gab. }\end{array}$ & $\begin{array}{c}\text { Vaucouleurs } \\
\text { (de) Prudence }\end{array}$ & 50000 & 30000 & Président \\
\hline
\end{tabular}

L'écart le plus net s'observe dans le cas Panou-Deurbroucq : on voit que la dot de 80000 livres apportée par Marie-Adrienne Deurbroucq, la fille d'un grand négociant nantais, rembourse aisément la charge de maître aux comptes acquise deux ans plus tôt par son nouvel époux, Jacques Louis Panou de Faymoreau. Au-delà du remboursement de l'office, la dot a permis ici au marié de s'enrichir considérablement comme en témoigne l'extrait suivant : "Le seigneur de Faymoreau comprit qu'une pareille demoiselle et une pareille dot lui conviendraient parfaitement bien. Mr de la Gourtière ${ }^{58}$ son cousin [qui avait épousé deux ans plus tôt une sœur de Marie-Adrienne Deurbroucq] vanta son château, ses richesses, ses vassaux et le mariage se conclut ${ }^{59}$. "L'alliance avec un magistrat pouvait se payer ainsi au prix fort surtout pour les familles qui tiraient leur fortune du commerce et qui désiraient progresser dans l'échelle des dignités.

\section{La rentabilité d'un office aux comptes}

On estime généralement que l'achat d'un office à la Chambre des comptes constituait un placement financier de rapport très convenable (5\%), nettement supérieur à la plupart des investissements fonciers (à l'exception des prés, vignes et des marais), mais à longue échéance seulement

58. Jacques François de la Gourtière, conseiller maître à la Chambre des comptes, mariée à Anne Deurbroucq. Il semblerait que la parenté entre la famille François de la Gourtière et Panou de Faymoreau se fasse par la famille François du Temps.

59. Extrait des Mémoires inédits de Pierre Panou de Faymoreau, collection privée. 
puisqu'il fallait rembourser le capital nécessaire à l'acquisition de l'office. Jean Meyer, dans son étude sur la noblesse bretonne, a ainsi donné l'exemple du maître aux comptes Jean-Baptiste Chotard de la Loierie, dont l'office rapporte, entre 1746 et 1776, un revenu annuel oscillant entre 2000 et 3000 livres ${ }^{60}$. Le magistrat, grâce à sa charge, disposait en effet de différents types de revenus, à savoir les gages, les épices, divers droits et les exemptions d'impôts pouvant être assimilées à un revenu supplémentaire.

Si l'on s'appuie sur notre échantillon de Poitevins, on peut prendre le cas de l'office de correcteur qui a été détenu tour à tour par Jean Victor Gabriel Chauvière de la Pagerie puis par son gendre Pierre Jacques Letard de la Bouralière. Si l'on en croit ce qu'écrit le premier, cette charge lui rapportait chaque année, 1200 livres « bien payées les trois termes par ans et en outre soixente dix huit livres tous les ans d'antrée et d'ailleurs sans compter tous les ans quelques réceptions d'officiers présidents généraux des finences encore soixante douze livres 16 sols " soit 1350 livres $^{61}$. Les informations fournies par le second conduisent à revoir ce chiffre à la baisse. Elles sont présentées dans un document de 1790 intitulé " Livre contenant par détail l'état de ma fortune actuelle et de celle de ma femme, tant en fonds qu'en rentes ${ }^{62}$ " et sont mêlées à des estimations de rentes foncières.

D'après cette comptabilité, l'office lui rapporte annuellement " dixième et capitation déduits " la somme de 1010 livres. Sur cette somme, il doit payer plusieurs rentes se montant à 834 livres que ses prédécesseurs (Guitton et Chauvière) avaient contractées pour le payement de l'office. Letard avait auparavant noté dans une marge de son " livre contenant la depense de notre menage en tout genre ${ }^{63}$ " que le 4 septembre " j'ai envoyé une lettre de change de $140 \mathrm{lt} 7 \mathrm{~s}$ à Mr Chaillou greffier de la Chambre des comptes de Nantes; laquelle jointe à 381 lt que mondit sr Chaillou touchera pour moi chés Mr Moreau pour le terme de noël de mes gages de 1789 et 26 lt qu'il a entre les mains à moi appartenan, forme un capital de 547 lt $7 \mathrm{~s}$ que je l'ai chargé de payer à mon acquit au sr Guyton de la Rairie, pour l'arrerage d'une rente que je lui dois, échue du 19 janvier dernier. Payé 547 It $7 \mathrm{~s}$ ». La rente des 547 livres est reportée dans ses comptes de novembre (voir tableau ci-dessous). Ce sont donc seulement 176 livres que Letard perçoit sur son office. Mais il est vrai qu'il était un officier peu assidu. Il rajoute pourtant à la fin de ses comptes, sans donner de chiffres ${ }^{64}$, qu'il lui est dû sur sa charge " les termes de St Jean et de Noël qui va écheoir; sans compter deux années arrierées ". L'absence au semestre n'entravait donc pas le payement des épices, c'est en tout cas ce que nous laisse penser Letard $^{65}$.

60. MeYER, Jean, La Noblesse bretonne..., op. cit., p. 177.

61. Arch. dép. de la Vienne, J 140.

62. Arch. dép. de la Vienne, J 149.

63. Arch. dép. de la Vienne, J 148.

64. Sauf la mention en septembre 1790 des 381 livres dues sur les gages de Noël 1789.

65. De même, le président Chéreil qui, dans une lettre au procureur général, n'hésitait pas réclamer sa part des épices malgré son défaut de service. Arch. dép. de Loire- 
Les Poitevins à la chambre des comptes de Bretagne au XVIII ${ }^{\mathrm{e}}$ siècle

Tableau 8 - Comptabilité concernant l'office de correcteur de PierreJacques Letard de la Bouralière pour l'année 1790

\begin{tabular}{|l|c|}
\hline $\begin{array}{l}\text { "Plus la charge de conseiller correcteur en la chambre des comptes de } \\
\text { Bretagne me rapporte annuellement, dixième et capitation déduits " }\end{array}$ & 1010 lt \\
\hline $\begin{array}{l}\text { "Sur quoi je dois une rente de } 605 \text { lt laquelle se réduit vingtiêmes retenus } \\
\text { a } 547 \text { lt environs (laditte rente échoit le } 19 \text { janvier) " }\end{array}$ & 547 lt \\
\hline $\begin{array}{l}\text { "Plus une autre de } 115 \text { lt payable le } 14 \text { fevr laquelle se réduit a } 102 \text { lt a } \\
\text { peuprès " }\end{array}$ & 102 lt \\
\hline "Plus une autre de cent livres, échoiant le 20 Xbre, elle se réduit à 89 lt " & 89 lt \\
\hline "Plus une autre de 96 lt sans retenue due au Sanitat de Nantes " & 96 lt \\
\hline \multicolumn{1}{|c|}{ Total } & 834 lt \\
\hline $\begin{array}{l}\text { "Otés 834 lt de } 1010 \text { lt, restent cent soixante seize livres. Il m'est du sur ma charge, les } \\
\text { termes de St Jean et de Noël qui va écheoir; sans compter deux années arrierées. " }\end{array}$ \\
\hline
\end{tabular}

Au total, la charge lui procure un revenu non négligeable malgré les anciennes créances à régler. En cas d'achat comptant, l'amortissement d'un office pouvait être très rapide. C'était donc un type d'investissement particulièrement favorable aux fortunes moyennes. Ce rapport correct explique que pour la plupart des magistrats il y avait bien un avant et un après le passage à la Chambre des comptes, bien qu'une distinction doive être faite entre les officiers.

Si l'on se fie aux quatre successions retrouvées, on observe que les fortunes laissées par deux auditeurs et un greffier en chef tournent autour de 200000 livres confortant l'idée d'appartenance de ces magistrats à la strate supérieure de la moyenne bourgeoisie ${ }^{66}$. Seule la succession de l'auditeur Pierre II Rochard de Landebergère paraît bien maigre avec seulement 63500 livres.

Quant aux deux successions reconstituées du maître aux comptes Jacques Louis Panou de Faymoreau et du président de Duval de Chassenon, elles se situent à un niveau bien supérieur. Le premier est en passe, à la veille de la Révolution, de devenir très fortuné (en partie grâce à la dot de son épouse). Il peut alors posséder un patrimoine estimé à 500000 livres. Quant à la famille des présidents Duval, déjà aisée avant son entrée à la Chambre des comptes, elle est à la tête d'une fortune estimée à 900000 livres lorsque le président de Chassenon quitte la compagnie en 1782. Il apparaît alors comme l'un des plus riches seigneurs du Poitou. Si l'on croit le portrait que donne de lui le baron de Frénilly dans ses Mémoires, le Président de Chassenon s'attira, en dépit de son immense fortune, une réputation d'avare de comédie : « Le gros président de Chassenon était président honoraire de

Atlantique, 2 E 1383.

66. Les successeurs de l'auditeur Jacques Joseph Jannet se partagent 180000 livres en 1776, ceux de l'auditeur Louis Charles Edouard Voyneau se partagent 232805 livres en 1787, et la succession du greffier en chef Jean Biaille vaut au moins 200000 livres en 1768 . 
la Chambre des comptes de Nantes, fort épais, fort riche et fort avare, mais de cette avarice, honteuse et fastueuse à la fois, où Molière aurait trouvé cent choses à prendre. Il faisait des peignoirs avec les vieilles jupes de sa fille en attachant à son col ce qu'elle avait attaché à sa ceinture ${ }^{67}$. "

\section{Deux destinées familiales singulières}

Grâce aux sources dont nous disposons, deux destins d'officier sont bien connus : l'un, celui de la famille Biaille de Lengibaudière, illustre un rare cas d'échec; l'autre, celui de la famille Cardin, présente un exemple de réussite lente et obstinée telle qu'il s'en produisait sans doute beaucoup à la Chambre des comptes.

\section{La famille Biaille de Lengibaudière, de l'honneur au déshonneur}

Jean-Louis Biaille de Lengibaudière est né en 1734. Son père, Jean, est alors greffier en chef de la Chambre des comptes de Bretagne depuis peu et son oncle Louis Jacques Biaille de la Milletière y sera reçu en tant qu'auditeur en 1748. Issu de la bourgeoisie d'offices poitevine, l'homme semble avoir un avenir assuré; sa famille étant une des plus aisées de la région $^{68}$. En 1756 et 1757 , alors licencié ès lois, il passe par Paris et Nantes, occupé à suivre les audiences. Après ses études et de retour dans sa province, Jean-Louis Biaille songe à s'établir et à se procurer une situation. Alors avocat et bientôt sénéchal de la principauté de Talmont, il épouse le 3 février 1761 Marie-Thérèse Mercier de Langevinière, fille d'un procureur des Sables-d'Olonne, d'une famille très riche, qui lui apporte la somme de 40000 livres. Quant à lui, il reçoit de son père une dot de 50000 livres qui comprend l'office de greffier en chef ${ }^{69}$. Tout semble assuré. Sa femme lui donne une fille, Marie Jeanne Prudence, née le 13 septembre 1762 à Talmont, et un fils Jean-Louis Marie, né le 7 octobre 1763 à Talmont, mais qui ne survit pas. Pourtant la lente ascension sociale commencée par ses ancêtres va se briser. Son amitié avec un ami de son père, Louis Auguste Barraud seigneur de la Rivière, suscite la jalousie d'un des hommes les plus puissants de la région, Jean-Baptiste Jacques Daniel de Loynes marquis de la Coudraye, gouverneur des ville et château de Fontenay-le-Comte et cousin par sa femme du seigneur de la Rivière. Il voit en effet d'un mauvais œil ce dernier vendre ses terres à Jean-Louis Biaille de Lengibaudière.

Se sachant malade, Louis-Auguste Barraud, ayant peu d'affection pour sa famille, avait en effet préféré vendre au fils de son ami Jean Biaille ses terres des Granges et de la Rivière. Ces ventes contenaient toutefois des vices de forme sur lesquels s'appuie le marquis de la Coudraye pour s'op-

67. FRÉNILLY, Baron de, Souvenirs du Baron de Frénilly, pair de France, Paris, 1908, p. 111 112.

68. MallLaud, Jean, Notes généalogiques, famille Biaille, tome 11.

69. Arch. dép. de Vendée, 1 E 683. 
poser à la transmission des biens. Après un procès retentissant qui passionne l'opinion de la province, voire au-delà, Jean-Louis Biaille, ainsi que ses parents, sont emprisonnés à la Conciergerie et il est pour sa part jugé par le parlement de Paris pour faux et escroquerie. Par le verdict prononcé le 3 mars 1769, il est déchu de toutes ses charges ${ }^{70}$ et est condamné à cinq années de galères qu'il passe au bagne de Brest. C'est le seul cas d'officier membre d'une cour souveraine ainsi condamné à Brest. S'il avait été membre d'un parlement on lui aurait certainement réservé une cellule dans une forteresse plus ou moins éloignée mais pour un greffier en chef de la Chambre des comptes, on n'a pas les mêmes égards ${ }^{71}$.

Sur le registre des matricules de Brest, Jean-Louis Biaille ne bénéficie pas de traitement spécifique. Il porte le numéro 3318 et est marqué sur l'épaule par l'exécuteur de la haute justice d'un fer chaud des initiales GAL. Arrivé le 13 juin 1769 dans la chaîne qui va de Paris à Brest, il en ressort exactement cinq ans plus tard, le 3 mars 1774 . On perd alors sa trace jusqu'au début de l'année 1777, date à laquelle il revient s'établir aux Sables-d'Olonne. Veuf, sa femme étant morte à Paris à la fin de l'année 1775, il retrouve sa fille unique, mais déshonoré par les cinq années au bagne, il souhaite désormais ne voir personne ${ }^{72}$.

Commence alors la seconde partie de sa vie qui tranche radicalement avec la première. Il se remarie le 11 juin 1783 avec Elisabeth Leroux, dite la Musse, "fille naturelle d'une femme débauchée et d'un soldat " et veuve d'un marin mort en mer, dont il avait déjà eu deux enfants. La rupture avec le passé est complète. Collinet, un observateur sablais, note dans son journal :

" [Elisabeth Leroux] allait avec d'autres courir en les campagnes pour vendre des sardines et autres poissons. Ce fut alors qu'elle commença à connaître le sieur Lengibaudière, sénéchal alors de Talmont, restant au château dudit lieu, où elle faisait ses commissions pour les Sables. La Musse avait épousé Jacques Mouroit des Sables, matelot ivrogne et mauvais marin, de qui elle a eu à souffrir pendant sept ans qu'elle est restée avec lui, les plus rudes traitements. Lengibaudière, à son arrivée de Brest, trouve sa mère morte, se retire aux Sables, prend la Musse pour gouvernante qui, un an après, accouche d'une fille [...]. Il est bon d'observer que ledit Lengibaudière avait épousé une Mercier de Langevinière, très riche, de qui il a eu une fille qui est actuellement avec lui. Lengibaudière conduisait la Musse à Nantes, où il l'habillait en demoiselle. Elle était de brunes piquantes, qui sans être belle, avait en son minois et en ses yeux un air attrayant et honnête, sans

70. Selon son factum, les officiers de la Chambre des comptes étaient solidaires, ce qu'attesta Fleury, le premier commis au greffe. Au contraire, le factum de Loynes de la Coudraye rapporte que la compagnie n'approuvait pas l'attestation de Fleury.

71. Remarque suggérée par Philippe Jarnoux, professeur d'histoire moderne à l'université de Brest, dans " D'Anjou en Bretagne : mobilités géographiques et mobilités sociales ", dans HAUDRÈRE, Philippe (dir.), Pour une histoire sociale des villes. Mélanges offerts à Jacques Maillard, Rennes, 2006, p. 217-218.

72. ColLinEt, André, Les Sables-d'Olonne au temps de la grande pêche. Les manuscrits de Collinet (1739-1782), La Mothe-Achard, 2003, p. 202. 
coquetterie. [...] Lengibaudière père étant décédé, le fils a épousé la Musse. Quoique cette femme, se soit vue à la tête d'une fortune de cent mille écus, elle n'a pas élevé son ton humble ${ }^{73}$."

Lors de son mariage en 1783, aucun membre de sa famille n'est présent, et en 1785 le parrain de sa fille est Nicolas Cochon, un boulanger, la marraine ne sait pas signer et cette enfant épousera quelques années plus tard un modeste greffier de juge de paix. Ses deux autres filles épousèrent pour l'une un musicien militaire en l'an XIII, fils d'un maçon, et l'autre un receveur de l'enregistrement de Machecoul en l'an XII. Son seul héritier, Henri-Denis (1789-1820), épouse une lingère en 1813 ${ }^{74}$. On devine facilement le déclassement de celui qui aurait pu être anobli définitivement à partir de 1781 s'il n'avait été condamné, et qui pouvait penser assurer à sa descendance des positions solides ${ }^{75}$.

\section{La famille Cardin, une progression méthodique}

L'office de greffier en chef de Jean-Louis Biaille de Lengibaudière avait été récupéré en 1769 par Jean Cardin. La famille Cardin avait vu se succéder depuis le milieu du $\mathrm{XvI}^{\mathrm{e}}$ siècle quatre générations de marchands bouchers qui pratiquaient leur profession dans le faubourg du Marchou à Fontenayle-Comte. Grâce aux capitaux accumulés, Jean Cardin put envoyer, vers 1677 , son fils prénommé Jean, dans un des collèges de Fontenay-le-Comte (vraisemblablement celui des Jésuites). Après trois ou quatre ans passés dans cette institution, ce dernier entra chez un notaire pour y devenir clerc. Il en sortit en 1689 et en 1695 il acheta une étude à Fontenay-le-Comte ${ }^{76}$. En 1709, il cumula son office de notaire avec la charge de greffier des Eaux et Forêts de Fontenay-le-Comte jusqu'à sa mort en 1716. Son fils, lui aussi prénommé Jean, né en 1708 à Fontenay, suivit également le cursus collégial et accomplit en plus trois ans dans une université afin d'obtenir le grade d'avocat puisqu'on le trouve " homme de loy " et avocat au parlement de Bretagne. En 1759, il est assesseur à Fontenay-le-Comte et dans le même temps obtient un office anoblissant d'avocat secrétaire ordinaire du roi de Pologne Stanislas, fonction tout à fait honorifique. En 1764, il n'est plus assesseur mais devient l'année suivante échevin de la capitale bas-poitevine. À 60 ans, sa carrière est déjà bien remplie mais il réussit en 1769, grâce à ses relations familiales et à la protection de grands seigneurs, à acquérir un office de greffier en chef à la Chambre des comptes de Bretagne qui couronne donc une belle carrière. Un premier pas est posé vers la noblesse. Son mariage avec Jeanne Gabrielle Merland, la fille d'un échevin de Fontenayle-Comte, relève de l'anecdotique. Son fils aîné, Jean-François Aimé Cardin,

73. Collinet, André, Les Sables et la guerre de Vendée. Les manuscrits de Collinet (17881804), La Mothe-Achard, 2003, p. 159-160.

74. ChASSIN du GuERNY, Recueil de filiations bas-poitevines, Montauban, 1961, p. 52-53. Henri Denis Biaille eut un fils en 1814, Moïse Henri Charles, propriétaire à Talmont.

75. JARnoux, Philippe, " D’Anjou en Bretagne... ", art. cit., p. 217-218.

76. MaIllaud, Jean, Notes généalogiques, famille Cardin, tome 5. 
alias Jules François-Aimé, né en 1756 à Fontenay, poursuit lui aussi une licence en droit et grâce aux relations de son père, il acquiert en 1778, à l'âge de 22 ans, un office d'auditeur à la Chambre des comptes. Ainsi, la famille voit avec certitude son entrée dans la noblesse, elle qui pratiquait, un siècle auparavant, le commerce de la boucherie dans un faubourg populaire de Fontenay-le-Comte. Quoique stoppée par la Révolution, l'ascension est réelle mais elle ne s'arrête pas là puisque Jean-François Aimé épouse en 1793 Marie-Julie Louvart de Pontlevoye, d'une famille de noblesse d'épée qui faisait remonter sa noblesse jusqu'en 1388! Et même si la Révolution ne lui a pas permis d'atteindre la noblesse pleine et entière, la rapide montée sociale de cette famille n'en est que plus frappante.

Si leur comportement social semble similaire tout au long du XVIII ${ }^{\mathrm{e}}$ siècle, l'attitude de ces officiers n'a pas été la même face à la Révolution. Certains ont vu avec enthousiasme l'instauration d'une monarchie constitutionnelle comme l'auditeur Jacques Joseph Marie Bourasseau qui se fait élire député du Maine-et-Loire dès la création des départements sous la Constituante. Le correcteur Pierre Jacques Letard de la Bouralière est quant à lui nommé président du district de Chinon grâce à la protection de son beau-père. Les autres officiers retournent dans leurs domaines à la campagne, loin des révoltes, ou dans leur appartement en ville non sans pester contre la plantation des arbres de la liberté comme l'un des membres de la famille Pichard. Ils profitent " de leurs loisirs forcés " pour se consacrer à l'amélioration de l'exploitation de leurs fermes et à la gestion de leur patrimoine comme l'auditeur Jean-François Aimé Cardin qui se qualifie d'" agriculteur $^{77}$ ". Certains d'entre eux n'hésitent pas à arrondir leur patrimoine en acquérant les biens ecclésiastiques devenus nationaux comme le Président de Chassenon ou le correcteur Chauvière de la Pagerie.

À notre connaissance, un seul ancien magistrat émigre, Jacques Louis Panou de Faymoreau, issu d'une famille engagée depuis trois générations déjà dans le système seigneurial. Se découvrant des affaires embrouillées en Flandre (il avait épousé la fille d'un important négociant de Nantes), il quitte le pays en octobre 1791 avec ses deux fils aînés. Après la défaite de l'armée des Princes, l'ancien conseiller maître gagne Hambourg pour y retrouver des relations et envoie ses deux fils se battre en Vendée. Le soulèvement royaliste vendéen constitue en effet un tournant pour ces anciens magistrats. Les familles Pichard, Cossin, Soulard, Arnault, Boutillier, entre autres, ont pris fait et cause pour le soulèvement royaliste. D'ailleurs, l'ancien auditeur Louis Charles Arnault prend une part active dans le soulèvement et meurt en 1794. La famille Boutillier paye également cher sa participation aux combats.

Le coup d'État du 18 brumaire vient bouleverser une nouvelle fois la vie des magistrats qui s'étaient jusqu'alors tenus à l'écart. Letard et le président de Chassenon font d'abord partie du conseil municipal de Poitiers

77. Arch. dép. de Vendée, 3 E 37/219. 
où ils sont nommés par le préfet, respectivement en 1800 et 1804. Letard, qui jouit d'un crédit suffisant, est nommé peu de temps après conseiller en la Cour impériale de Poitiers dès son établissement ${ }^{78}$. Le président de Chassenon place ses enfants dans de hautes fonctions administratives ${ }^{79}$. Le seigneur de Faymoreau, revenu de son périple en Allemagne et très proche du préfet vendéen, accepte à partir de 1808 la modeste fonction de maire de sa commune. S'ils ne semblent pas être des admirateurs forcenés du nouveau régime, ils lui doivent en tout cas leur retour dans les honneurs. Toutefois, leur ralliement de coeur à la restauration des Bourbons en 1814 et leur silence pendant les Cent Jours sont significatifs. Letard est alors anobli ${ }^{80}$ et la carrière du fils aîné du président de Chassenon prend son véritable essor à partir de 1815; il fut plus tard comblé d'honneurs par Charles x et fait vicomte en 1824. Seul le seigneur de Faymoreau, dans son obscur château, fait figure d'oublié alors que ses relations lui auraient permis d'entreprendre une nouvelle carrière.

Aujourd'hui, les tombes toujours existantes du président de Chassenon et du maître Jacques Louis Panou de Faymoreau rappellent encore au passant la présence de ces deux magistrats à la Chambre des comptes de Bretagne.

78. LÉVÊQUE Guillaume, Dion Emmanuel, JAHAN Sébastien, Grands notables du Premier Empire, Vienne-Paris, 2000, p. 187-189.

79. Ibidem, p. 132-134.

80. La mort de son fils aîné à Dresde précipita Pierre Jacques Letard de la Bouralière dans les bras des Bourbons. 
Annexe - Les officiers d'origine poitevine de la Chambre des comptes de Nantes au XVII ${ }^{e}$ siècle

\begin{tabular}{|c|c|c|c|c|}
\hline Nom & Office & $\begin{array}{l}\text { Date de } \\
\text { réception }\end{array}$ & $\begin{array}{c}\text { Date de départ } \\
\text { (mode de départ) }\end{array}$ & Successeur \\
\hline $\begin{array}{l}\text { François-Gabriel DUVAL, } \\
\text { sieur de la Vergne }\end{array}$ & Président & $05-12-1759$ & $\begin{array}{l}\text { 11-09-1762 } \\
\text { (décès) }\end{array}$ & $\begin{array}{l}\text { François-Gabriel- } \\
\text { Joseph-Henri DUVAL } \\
\text { Poitou }\end{array}$ \\
\hline \begin{tabular}{|l|} 
François-Gabriel- \\
Joseph-Henri DUVAL, \\
sieur de Chassenon
\end{tabular} & Président & $17-01-1763$ & $\begin{array}{c}09-02-1782 \\
\text { (résignation) }\end{array}$ & $\begin{array}{l}\text { Marie-Jean-Philippe } \\
\text { PASCAUD Aunis }\end{array}$ \\
\hline $\begin{array}{l}\text { Pierre-Thomas } \\
\text { CICOTEAU, sieur } \\
\text { d'Ardennes }\end{array}$ & Maître & $16-03-1733$ & $\begin{array}{l}\text { 30-11-1735 } \\
\text { (décès) }\end{array}$ & $\begin{array}{l}\text { Pierre MOYSAN } \\
\text { Evêché de Nantes }\end{array}$ \\
\hline $\begin{array}{l}\text { Nicolas-Joseph } \\
\text { ROCQUAND, sieur de } \\
\text { Pontbureau } \\
\end{array}$ & Maître & $10-02-1749$ & $\begin{array}{l}\text { 15-04-1771 } \\
\text { (résignation) }\end{array}$ & $\begin{array}{l}\text { Pierre JOLLIVET } \\
?\end{array}$ \\
\hline \begin{tabular}{|l|} 
François-Gabriel- \\
Joseph-Henri DUVAL, \\
sieur de Chassenon
\end{tabular} & Maître & 09-09-1758 & $\begin{array}{l}17-01-1763 \\
\text { (résignation) }\end{array}$ & $\begin{array}{l}\text { Jacques-François } \\
\text { MAUVILLAIN Évêché } \\
\text { de Nantes }\end{array}$ \\
\hline \begin{tabular}{|l|} 
Jean-Baptiste-Marc- \\
Pascal ROBERT, sieur de \\
la Levraudière
\end{tabular} & Maître & $05-03-1770$ & $\begin{array}{l}\text { Suppression de } \\
\text { la Chambre }\end{array}$ & \\
\hline \begin{tabular}{|l} 
Jacques-Louis PANOU, \\
sieur de Faymoreau
\end{tabular} & Maître & $10-11-1770$ & $\begin{array}{l}\text { Suppression de } \\
\text { la Chambre }\end{array}$ & \\
\hline $\begin{array}{l}\text { Marin-Jean-Baptiste } \\
\text { BOUTILLIER, sieur de } \\
\text { la Chèze }\end{array}$ & Maître & $14-08-1781$ & $\begin{array}{l}\text { Suppression de } \\
\text { la Chambre }\end{array}$ & \\
\hline $\begin{array}{l}\text { Joseph ROCQUAND, } \\
\text { sieur de Pontbureau }\end{array}$ & Correcteur & $18-08-1746$ & $\begin{array}{l}\text { 24-02-1761 } \\
\text { (décès) }\end{array}$ & $\begin{array}{l}\text { Jean-Baptiste-Michel- } \\
\text { René PREGENT } \\
\text { Anjou }\end{array}$ \\
\hline $\begin{array}{l}\text { Jean-Victor-Gabriel } \\
\text { CHAUVIERE, sieur de la } \\
\text { Pagerie }\end{array}$ & Correcteur & $19-01-1763$ & $\begin{array}{l}27-09-1785 \\
\text { (résignation) }\end{array}$ & $\begin{array}{l}\text { Pierre-Jacques LETARD } \\
\text { Poitou }\end{array}$ \\
\hline $\begin{array}{l}\text { Pierre Jacques LETARD, } \\
\text { sieur de la Bouralière }\end{array}$ & Correcteur & $14-09-1785$ & $\begin{array}{l}\text { Suppression de } \\
\text { la Chambre }\end{array}$ & \\
\hline $\begin{array}{l}\text { Pierre I ROCHARD, sieur } \\
\text { de Landebergère }\end{array}$ & Auditeur & $17-02-1702$ & $\begin{array}{l}\text { 02-01-1726 } \\
\text { (résignation) }\end{array}$ & $\begin{array}{l}\text { François TREBILLARD } \\
\text { Evêché de Nantes }\end{array}$ \\
\hline $\begin{array}{l}\text { Louis CICOTEAU, sieur } \\
\text { de la Touche }\end{array}$ & Auditeur & $12-11-1716$ & 19-01-1737 & $\begin{array}{l}\text { Jacques-Joseph } \\
\text { JANNET Poitou }\end{array}$ \\
\hline $\begin{array}{l}\text { Réné-Louis VOYNEAU, } \\
\text { sieur du Plessis- } \\
\text { Mauclerc }\end{array}$ & Auditeur & 13-09-1719 & $\begin{array}{l}27-11-1741 \\
\text { (résignation) }\end{array}$ & $\begin{array}{l}\text { Louis-Charles-Edouard } \\
\text { VOYNEAU Poitou }\end{array}$ \\
\hline $\begin{array}{l}\text { Pierre-Jacques ROBERT, } \\
\text { sieur des Essertons }\end{array}$ & Auditeur & $07-05-1721$ & $\begin{array}{l}\text { 19-04-1739 } \\
\text { (décès) }\end{array}$ & $\begin{array}{l}\text { Paul-François BABIN } \\
\text { Poitou }\end{array}$ \\
\hline $\begin{array}{l}\text { Louis-Venant } \\
\text { CICOTEAU, sieur de } \\
\text { Linières }\end{array}$ & Auditeur & $18-02-1724$ & $\begin{array}{c}1729 \\
\text { (décès) }\end{array}$ & $\begin{array}{l}\text { Jean-Baptiste-Julien } \\
\text { MORISSET Évêché de } \\
\text { Nantes }\end{array}$ \\
\hline $\begin{array}{l}\text { Jude BADEREAU, sieur } \\
\text { de la Saminière }\end{array}$ & Auditeur & $31-03-1724$ & $\begin{array}{l}\text { 23-04-1741 } \\
\text { (décès) }\end{array}$ & $\begin{array}{l}\text { Grégoire TERRIEN } \\
\text { Evêché de Nantes }\end{array}$ \\
\hline $\begin{array}{l}\text { Jean-Jude BADEREAU, } \\
\text { sieur de la Caffinière }\end{array}$ & Auditeur & $18-01-1730$ & $\begin{array}{l}\text { 19-06-1730 } \\
\text { (décès) }\end{array}$ & $\begin{array}{l}\text { Armand-Gabriel- } \\
\text { François BADEREAU } \\
\text { Poitou }\end{array}$ \\
\hline $\begin{array}{l}\text { Armand-Gabriel- } \\
\text { François BADEREAU, } \\
\text { sieur du Boiscorbeau }\end{array}$ & Auditeur & $11-09-1730$ & $\begin{array}{c}17-02-1751 \\
\text { (résignation) }\end{array}$ & $\begin{array}{l}\text { Mathurin-Joseph } \\
\text { BABIN } \\
\text { Poitou }\end{array}$ \\
\hline
\end{tabular}


Guillaume PORCHET

\begin{tabular}{|c|c|c|c|c|}
\hline $\begin{array}{l}\text { Jacques-Joseph } \\
\text { JANNET, sieur de la } \\
\text { Jarrie }\end{array}$ & Auditeur & $19-01-1737$ & $\begin{array}{c}30-04-1757 \\
\text { (résignation) }\end{array}$ & $\begin{array}{l}\text { Pierre-Arthur } \\
\text { BERITAULT } \\
\text { Anjou }\end{array}$ \\
\hline $\begin{array}{l}\text { Paul-François BABIN, } \\
\text { sieur des Ardilliers }\end{array}$ & Auditeur & $05-05-1740$ & $\begin{array}{c}14-11-1778 \\
\text { (résignation) }\end{array}$ & $\begin{array}{l}\text { Jean-François-Aimé } \\
\text { CARDIN Poitou }\end{array}$ \\
\hline $\begin{array}{l}\text { Louis-Charles-Edouard } \\
\text { VOYNEAU, sieur du } \\
\text { Plessis-Mauclerc }\end{array}$ & Auditeur & $27-11-1741$ & $\begin{array}{l}\text { 12-09-1767 } \\
\text { (résignation) }\end{array}$ & $\begin{array}{l}\text { René-Jean RELIQUET } \\
\text { Evêché de Nantes }\end{array}$ \\
\hline $\begin{array}{l}\text { Jacques PANOU, sieur } \\
\text { de Faymoreau }\end{array}$ & Auditeur & $13-02-1744$ & $\begin{array}{c}\text { 19-12-1764 } \\
\text { (résignation) }\end{array}$ & $\begin{array}{l}\text { Paul RAZEAU } \\
\text { Évêché de Nantes }\end{array}$ \\
\hline $\begin{array}{l}\text { Louis-Jacques BIAILLE, } \\
\text { sieur de la Milletière }\end{array}$ & Auditeur & $20-09-1748$ & $\begin{array}{c}30-12-1769 \\
\text { (résignation) }\end{array}$ & $\begin{array}{l}\text { Maximilien ARNAULT } \\
\text { Poitou }\end{array}$ \\
\hline $\begin{array}{l}\text { Pierre II ROCHARD, } \\
\text { sieur de Landebergère }\end{array}$ & Auditeur & $05-12-1748$ & $\begin{array}{l}\text { 10-1768 } \\
\text { (décès) }\end{array}$ & $\begin{array}{l}\text { Claude-René BLOUIN } \\
\text { Anjou }\end{array}$ \\
\hline $\begin{array}{l}\text { Charles-Laurent } \\
\text { BOUHIER, sieur de } \\
\text { Beauregard }\end{array}$ & Auditeur & 21-01-1750 & $\begin{array}{l}11-09-1775 \\
\text { (résignation) }\end{array}$ & $\begin{array}{l}\text { Jean-Baptiste-GAUDIN } \\
\text { Anjou }\end{array}$ \\
\hline $\begin{array}{l}\text { Mathurin-Joseph BABIN, } \\
\text { sieur de la Chevallerie }\end{array}$ & Auditeur & $17-02-1751$ & $\begin{array}{c}13-09-1779 \\
\text { (résignation) }\end{array}$ & $\begin{array}{l}\text { Charles-Lezin COSSIN } \\
\text { Poitou }\end{array}$ \\
\hline $\begin{array}{l}\text { Maximilien ARNAULT, } \\
\text { sieur de la Motte }\end{array}$ & Auditeur & $30-12-1769$ & 5-12-1789 (décès) & (Pas de remplaçant) \\
\hline $\begin{array}{l}\text { Jean-François-Aimé } \\
\text { CARDIN, sieur des } \\
\text { Nouhes }\end{array}$ & Auditeur & $14-11-1778$ & $\begin{array}{l}\text { Suppression de } \\
\text { la Chambre }\end{array}$ & \\
\hline $\begin{array}{l}\text { Jacques-Joseph-Marie } \\
\text { BOURASSEAU, sieur de } \\
\text { la Renollière }\end{array}$ & Auditeur & $07-01-1779$ & $\begin{array}{l}\text { Suppression de } \\
\text { la Chambre }\end{array}$ & \\
\hline $\begin{array}{l}\text { Charles-Lezin COSSIN, } \\
\text { sieur de Belletouche }\end{array}$ & Auditeur & $13-02-1779$ & $\begin{array}{l}05-1784 \\
\text { (décès) }\end{array}$ & $\begin{array}{l}\text { Louis-Charles } \\
\text { ARNAULT Poitou }\end{array}$ \\
\hline $\begin{array}{l}\text { Marie-Augustin } \\
\text { PICHARD, sieur de la } \\
\text { Caillère }\end{array}$ & Auditeur & $02-12-1784$ & $\begin{array}{l}\text { 23-04-1786 } \\
\text { (décès) }\end{array}$ & $\begin{array}{l}\text { François-Augustin } \\
\text { PICHARD Poitou }\end{array}$ \\
\hline $\begin{array}{l}\text { Louis-Charles ARNAULT, } \\
\text { sieur de la Motte }\end{array}$ & Auditeur & $12-03-1785$ & $\begin{array}{l}\text { Suppression de } \\
\text { la Chambre }\end{array}$ & \\
\hline $\begin{array}{l}\text { François SOULARD, } \\
\text { sieur de la ROCHE }\end{array}$ & Auditeur & $23-06-1786$ & $\begin{array}{l}\text { Suppression de } \\
\text { la Chambre }\end{array}$ & \\
\hline $\begin{array}{l}\text { François-Augustin } \\
\text { PICHARD, sieur de la } \\
\text { Caillère }\end{array}$ & Auditeur & 03-08-1786 & $\begin{array}{l}\text { Suppression de } \\
\text { la Chambre }\end{array}$ & \\
\hline $\begin{array}{l}\text { Jean BIAILLE, sieur de } \\
\text { Lengibaudière }\end{array}$ & $\begin{array}{l}\text { Greffier en } \\
\text { chef }\end{array}$ & $20-12-1732$ & $\begin{array}{c}12-03-1761 \\
\text { (résignation) }\end{array}$ & $\begin{array}{l}\text { Jean-Louis BIAILLE } \\
\text { Poitou }\end{array}$ \\
\hline $\begin{array}{l}\text { Simon BOURDEAU, } \\
\text { sieur du Boislambert }\end{array}$ & $\begin{array}{l}\text { Greffier en } \\
\text { chef }\end{array}$ & 09-03-1750 & $\begin{array}{c}17-12-1770 \\
\text { (résignation) }\end{array}$ & $\begin{array}{l}\text { Louis-Marie ARNAULT } \\
\text { Poitou }\end{array}$ \\
\hline $\begin{array}{l}\text { Jean-Louis BIAILLE, } \\
\text { sieur de Lengibaudière }\end{array}$ & $\begin{array}{c}\text { Greffier en } \\
\text { chef }\end{array}$ & $12-03-1761$ & $\begin{array}{c}1765 \\
\text { (démission) }\end{array}$ & $\begin{array}{l}\text { Jean CARDIN } \\
\text { Poitou }\end{array}$ \\
\hline $\begin{array}{l}\text { Jean CARDIN, sieur } \\
\text { d'Anchais }\end{array}$ & $\begin{array}{l}\text { Greffier en } \\
\text { chef }\end{array}$ & $07-09-1769$ & $\begin{array}{l}\text { Suppression de } \\
\text { la Chambre }\end{array}$ & \\
\hline $\begin{array}{l}\text { Louis-Marie ARNAULT, } \\
\text { sieur de la Fauconnière }\end{array}$ & $\begin{array}{l}\text { Greffier en } \\
\text { chef }\end{array}$ & $17-12-1770$ & $\begin{array}{l}\text { 12-09-1780 } \\
\text { (décès) }\end{array}$ & $\begin{array}{l}\text { Jean-Baptiste-Alexis } \\
\text { PICHARD Poitou }\end{array}$ \\
\hline $\begin{array}{l}\text { Jean-Baptiste-Alexis } \\
\text { PICHARD, sieur de la } \\
\text { Blanchère }\end{array}$ & $\begin{array}{l}\text { Greffier en } \\
\text { chef }\end{array}$ & $19-07-1781$ & $\begin{array}{l}\text { Suppression de } \\
\text { la Chambre }\end{array}$ & \\
\hline
\end{tabular}




\section{RÉSUMÉ}

Au XVIII ${ }^{e}$ siècle, la Chambre des comptes de Bretagne a représenté pour les élites poitevines une opportunité d'ascension brève et rapide. Pour les familles de noblesse récente, elle permit un affermissement de leur condition et pour les familles bourgeoises, elle autorisa un anoblissement en deux générations successives. Les quarante magistrats poitevins ont possédé principalement des offices subalternes (auditeur, greffier en chef). Lorsque la Révolution éclate, seulement trois familles ont été anoblies sur les vingt-deux entrées depuis 1702.

\section{ABSTRACT}

During the $18^{\text {th }}$ Century, the Brittany's Audit Office was the theatre of a brief and rapid social promotion opportunity for the Poitevine elite. For the recently ennobled families, it allowed a strengthening of their condition and for the bourgeoisie, it authorized a two generations ennoblement. The forty Poitevin magistrates have mainly held minor offices (Commissioners of audits, clerks). At the outbreak of the French Revolution, among the twenty-two admissions that occurred since 1702, only three families had been ennobled. 